\title{
Novel meshless method for solving the potential problems with arbitrary domain
}

\author{
D.L. Young *, K.H. Chen, C.W. Lee \\ Department of Civil Engineering and Hydrotech Research Institute, National Taiwan University, Taipei 10617, Taiwan
}

Received 16 August 2004; received in revised form 8 March 2005; accepted 8 March 2005

Available online 23 May 2005

\begin{abstract}
In this article, a non-singular and boundary-type meshless method in two dimensions is developed to solve the potential problems. The solution is represented by a distribution of the kernel functions of double layer potentials. By using the desingularization technique to regularize the singularity and hypersingularity of the kernel functions, the source points can be located on the real boundary and therefore the diagonal terms of influence matrices are determined. The main difficulty of the coincidence of the source and collocation points then disappears. By employing the two-point function, the off-diagonal coefficients of influence matrices are easily obtained. The numerical evidences of the proposed meshless method demonstrate the accuracy of the solutions after comparing with the results of exact solution, conventional MFS and BEM for the Dirichlet, Neumann and mix-type boundary conditions (BCs) of interior and exterior problems with simple and complicated boundaries. Good agreements with exact solutions are observed.
\end{abstract}

(c) 2005 Elsevier Inc. All rights reserved.

Keywords: Non-singular; Meshless method; Conventional MFS; Singular fundamental solution; Double layer potential; Desingularization technique; Singularity; Hypersingularity; Kernel function; Mixed-type BC; Circulants

\section{Introduction}

During the last decade, scientific researchers have paid attention to the meshless methods in which the mesh or element is free. The meshless methods are the mesh reduction methods with no meshes requirements and only boundary nodes are necessary. The mesh reduction techniques possess great progresses to compete with the FVM, FEM and FDM as dominant numerical methods. Because neither domain nor surface meshing is required, the meshless methods have become very attractive for engineers in model

\footnotetext{
* Corresponding author. Tel./fax: +886223626114.

E-mail address: dlyoung@ntu.edu.tw (D.L. Young).
} 
creation, and important tools for scientific computing. Several meshless methods have been reported in the literature, for example, the smooth particle hydrodynamics (SPH) method [12], the element-free Galerkin (EFG) method [2], the reproducing kernel particle method (RKPM) [16], the method of fundamental solutions (MFS) [6,11,18-21], boundary knot method (BKM) [8,9], boundary collocation method (BCM) [3-7] and boundary node method (BNM) [22-24]. These methods are truly meshless, since no domain or boundary meshes are required.

The MFS has been extensively applied to solve some engineering problems [10,11,18-21]. It is a kind of meshless methods, since only boundary nodes are distributed. A comprehensive review of the MFS was given by Fairweather and Karageorghis [11]. The solution procedure makes use of the fundamental solutions, which satisfies the governing equation in the interested domain. To avoid the singularity problem, the solution is represented as a set of singular kernels or the single layer potentials on non-physical boundary (fictitious boundary). The kernel function is composed of two-point function which is one kind of the radial basis functions (RBFs). The independent variable of two-point function depends on point position only. A regular singularity-free formulation was obtained as a result, and achieving an attractive truly boundarytype and mathematically simple meshfree method. However because of the controversial artificial boundary (off-set boundary) outside the physical domain, the MFS has not become a popular numerical method. The meaning of off-set boundary is an auxiliary boundary to offset a distance from the real boundary. In general for real engineering problems especially for a complicated geometry, the off-set boundary distance is difficult to determine. The diagonal coefficients of influence matrices are divergent due to the point collocation when the off-set boundary approaches to the real boundary. Despite its gain in singularity free, the influence matrices become ill-posed matrices when the off-set boundary is far away from the real boundary. It results in an ill-posed problem since the condition number for the influence matrix becomes very large. The location of source and observation points is vital to the accuracy of the solution by implementing the conventional MFS.

An improved approach called the BKM or BCM was introduced very recently, by Chen and his coworkers [3-7], and Kang and his collaborators [14,15] as well as Chen and his co-workers [8,9]. Instead of using the singular fundamental solutions, the non-singular kernels were employed to evaluate the homogeneous solution. These methods dealt successfully with many kinds of problems and eliminated the wellknown drawback of ambiguous off-set boundary. The major differences in these meshless methods come only from the techniques used for the chosen non-singular kernels RBFs. However, the introduction of non-singular kernels may jeopardize the accuracy of the solutions as comparing with using the singular fundamental solutions. Another improved method is called the Hybrid boundary node method (Hybrid $\mathrm{BNM}$ ), which combines the moving least squares (MLS) interpolation scheme with the hybrid displacement variational formulation [23,24]. However, some integration is still needed as far as with the BNM or Hybrid BNM.

In these BKM and BCM references, the methods only worked well in regular geometry with the Dirichlet and Neumann BCs. Even though these methods can locate the source points on the physical boundary and use the non-singular kernels, there still accompanies some difficulty at the ill-posed problems. Therefore, the purpose of this paper is to develop a novel meshless method for solving the potential problems based on the potential theory as well as the desingularization of subtracting and adding-back technique [13,17] to regularize the singularity and hypersingularity of the kernel functions. The proposed method is to distribute the observation and source points on the coincident locations of the real boundary even using the singular kernels (double layer potentials) instead of non-singular kernels and still maintains the spirit of the MFS. The diagonal terms of the influence matrices can be derived by using the proposed technique. Also, the influence coefficients by numerical methods are compared with analytical solutions by using separable kernels [1] and circulants [4] for the circular domain. Finally, a new program of the novel meshless method is constructed to solve the Laplace problems subject to the Dirichlet, Neumann and mix-type problems. This includes continuous or discontinuous BCs with the smooth and non-smooth simple and complicated boundaries. 


\section{Formulation}

Consider a boundary value problem with a potential $\phi(x)$, which satisfies the Laplace equation as follows:

$$
\nabla^{2} \phi(x)=0, \quad x \in D
$$

subject to $\mathrm{BCs}$

$$
\begin{aligned}
& \phi(x)=\bar{\phi}, \quad x \in B_{1}, \\
& \psi(x)=\bar{\psi}, \quad x \in B_{2},
\end{aligned}
$$

where $\nabla^{2}$ is the Laplacian operator, $D$ is the domain of the problem. The boundary conditions are described as following: where $\psi(x)=\frac{\partial \phi(x)}{\partial n_{x}}$ and $B_{1}$ is the essential boundary (Dirichlet boundary) in which the potential is prescribed as $\bar{\phi} ; B_{2}$ is the natural boundary (Neumann boundary) in which the normal derivative is prescribed as $\bar{\psi}$; and $B_{1}$ and $B_{2}$ construct the whole boundary of the domain $D$ as well as the outside domain $D^{e}$ as shown in Fig. 1. The real physical problems for the Laplace equation contain potential flow problems, torsion bar problems, Stokes equations of the vorticity transport equations, etc. By employing the RBF technique $[8,18]$, the representation of the solution for interior problem can be approximated in terms of the strengths $\alpha^{j}$ of the singularities $s^{j}$ as

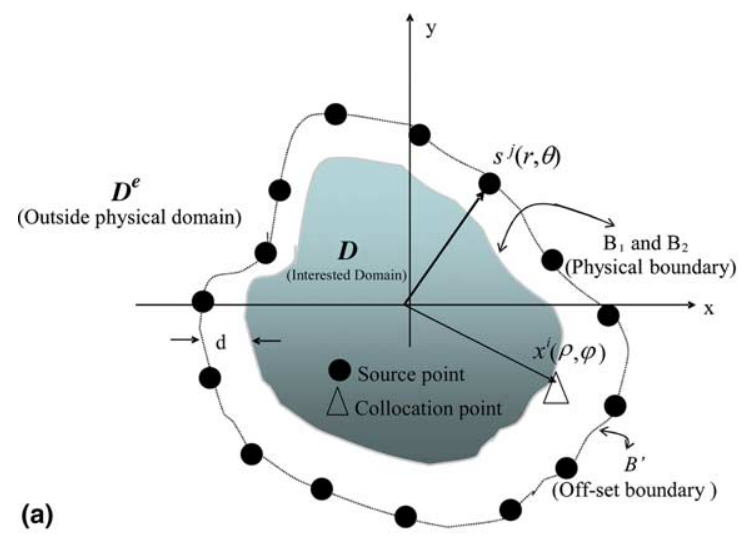

(a)

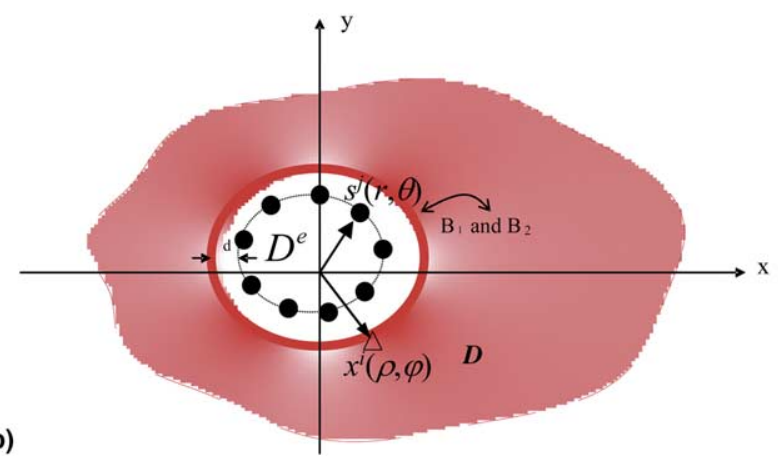

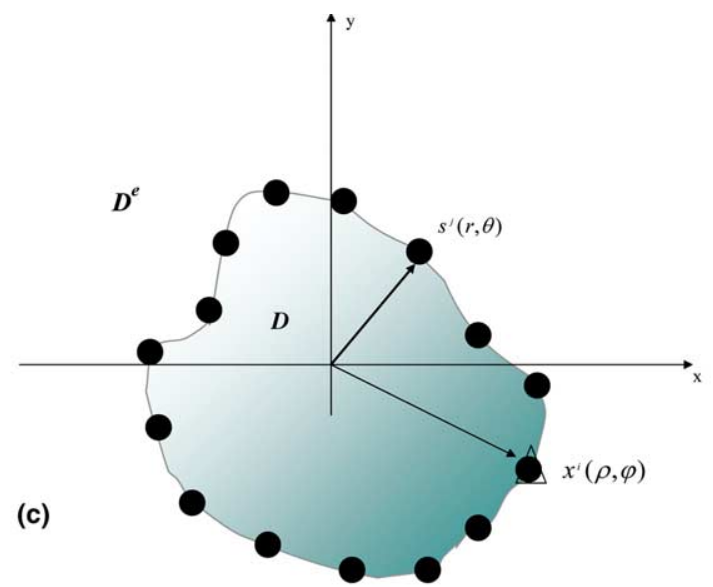

(d)

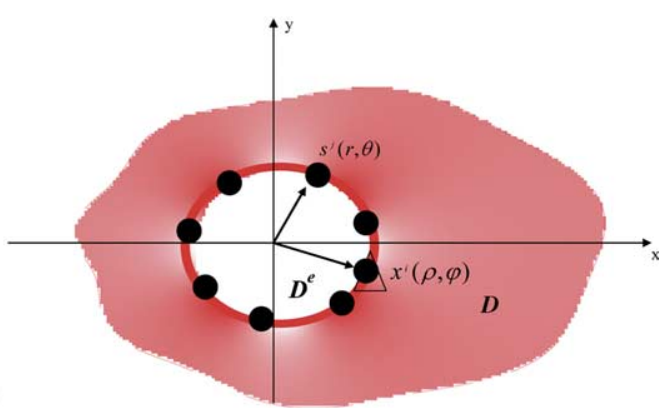

Fig. 1. The source point and observation point distributions and definitions of $r, \theta, \rho, \varphi$ by using the conventional MFS and the novel meshless method for the interior and exterior problems: (a) interior problem (MFS), (b) exterior problem (MFS), (c) interior problem (proposed method), (d) exterior problem (proposed method). 


$$
\begin{aligned}
& \phi\left(x^{i}\right)=\sum_{j=1}^{N} A^{(\mathrm{i})}\left(s^{j}, x^{i}\right) \alpha^{j}, \\
& \psi\left(x^{i}\right)=\sum_{j=1}^{N} B^{(\mathrm{i})}\left(s^{j}, x^{i}\right) \alpha^{j},
\end{aligned}
$$

where $A^{(\mathrm{i})}\left(s^{j}, x^{i}\right)$ is RBF in which the superscript (i) denotes the interior domain, $\alpha^{j}$ are the $j$ th unknown coefficients (strengths of the singularities), $s^{j}$ is $j$ th source point (singularity), $x^{i}$ is $i$ th observation point, $N$ is the numbers of source points and $B^{(\mathrm{i})}\left(s^{j}, x^{i}\right)=\frac{\partial A^{(i)}\left(s^{j}, x^{i}\right)}{\partial n_{x^{i}}}$. The coefficients $\left\{\alpha^{j}\right\}_{j=1}^{N}$ are determined so that $\mathrm{BC}$ is satisfied at the boundary points, $\left\{x^{i}\right\}_{i=1}^{N}$. In Fig. $1^{x^{i}}$ the distributions of source points and observation points are shown for the interior and exterior problems. The descriptions of the terminology of observation points, source points, field points, collocation points, boundary points, two-point function, off-set boundary and strength of singularity can also be found in $[4,6]$.

By collocating $N$ observation points to match with the BCs from Eq. (4) for Dirichlet problems and Eq. (5) for the Neumann problems, we have the following linear systems of the form

$$
\begin{aligned}
& {\left[\begin{array}{cccc}
a_{1,1} & a_{1,2} & \cdots & a_{1, N} \\
a_{2,1} & a_{2,2} & \cdots & a_{2, N} \\
\vdots & \vdots & \ddots & \vdots \\
a_{N, 1} & a_{N, 2} & \cdots & a_{N, N}
\end{array}\right]\left\{\alpha^{j}\right\}=\left[A^{(\mathrm{i})}\right]\left\{\alpha^{j}\right\}=\left\{\bar{\phi}^{(\mathrm{i})}\right\},} \\
& {\left[\begin{array}{cccc}
b_{1,1} & b_{1,2} & \cdots & b_{1, N} \\
b_{2,1} & b_{2,2} & \cdots & b_{2, N} \\
\vdots & \vdots & \ddots & \vdots \\
b_{N, 1} & b_{N, 2} & \cdots & b_{N, N}
\end{array}\right]\left\{\alpha^{j}\right\}=\left[B^{(\mathrm{i})}\right]\left\{\alpha^{j}\right\}=\left\{\bar{\psi}^{(\mathrm{i})}\right\},}
\end{aligned}
$$

where

$$
\begin{aligned}
& a_{i, j}=A^{(\mathrm{i})}\left(s^{j}, x^{i}\right), \quad i, j=1,2, \ldots, N, \\
& b_{i, j}=B^{(\mathrm{i})}\left(s^{j}, x^{i}\right), \quad i, j=1,2, \ldots, N
\end{aligned}
$$

For the mixed-type problems, a linear combination of Eqs. (6) and (7) is made to satisfy the mixed-type BCs. After solving the unknown density functions $\left\{\alpha^{j}\right\}_{j=1}^{N}$ with the linear algebraic solver, the solutions for the interested domain are calculated from the field equations (4) and (5).

Similarly for the exterior problems, we have

$$
\begin{aligned}
& \phi\left(x^{i}\right)=\sum_{j=1}^{N} A^{(\mathrm{e})}\left(s^{j}, x^{i}\right) \alpha^{j}, \\
& \psi\left(x^{i}\right)=\sum_{j=1}^{N} B^{(\mathrm{e})}\left(s^{j}, x^{i}\right) \alpha^{j},
\end{aligned}
$$

where the superscript of $A^{(\mathrm{e})}\left(s^{j}, x^{i}\right)$ denotes the exterior domain. After collocating $N$ observation points with the Dirichlet or Neumann BCs, we obtain 


$$
\begin{aligned}
& {\left[\begin{array}{cccc}
\bar{a}_{1,1} & \bar{a}_{1,2} & \cdots & \bar{a}_{1, N} \\
\bar{a}_{2,1} & \bar{a}_{2,2} & \cdots & \bar{a}_{2, N} \\
\vdots & \vdots & \ddots & \vdots \\
\bar{a}_{N, 1} & \bar{a}_{N, 2} & \cdots & \bar{a}_{N, N}
\end{array}\right]\left\{\alpha^{j}\right\}=\left[A^{(\mathrm{e})}\right]\left\{\alpha^{j}\right\}=\left\{\bar{\phi}^{(\mathrm{e})}\right\},} \\
& {\left[\begin{array}{cccc}
\bar{b}_{1,1} & \bar{b}_{1,2} & \cdots & \bar{b}_{1, N} \\
\bar{b}_{2,1} & \bar{b}_{2,2} & \cdots & b_{2, N} \\
\vdots & \vdots & \ddots & \vdots \\
\bar{b}_{N, 1} & \bar{b}_{N, 2} & \cdots & \bar{b}_{N, N}
\end{array}\right]\left\{\alpha^{j}\right\}=\left[B^{(\mathrm{e})}\right]\left\{\alpha^{j}\right\}=\left\{\bar{\psi}^{(\mathrm{e})}\right\} .}
\end{aligned}
$$

Similar procedures as the interior problems are undertaken to obtain the field solutions for the exterior problems. According to the dependence of the outward normal vectors in the two kernel functions for interior and exterior problems, their relationships are

$$
\begin{aligned}
& \begin{cases}A^{(\mathrm{i})}\left(s^{j}, x^{i}\right)=-A^{(\mathrm{e})}\left(s^{j}, x^{i}\right), & i \neq j, \\
A^{(\mathrm{i})}\left(s^{j}, x^{i}\right)=A^{(\mathrm{e})}\left(s^{j}, x^{i}\right), & i=j,\end{cases} \\
& \begin{cases}B^{(\mathrm{i})}\left(s^{j}, x^{i}\right)=B^{(\mathrm{e})}\left(s^{j}, x^{i}\right), & i \neq j, \\
B^{(\mathrm{i})}\left(s^{j}, x^{i}\right)=B^{(\mathrm{e})}\left(s^{j}, x^{i}\right), & i=j .\end{cases}
\end{aligned}
$$

The chosen RBFs in this study are the double layer potentials in the potential theory and were derived in Appendix A for the exterior problems or can be found in $[4,5]$ as

$$
\begin{aligned}
& A^{(\mathrm{e})}\left(s^{j}, x^{i}\right)=\frac{n_{k} y_{k}}{\bar{r}_{i j}^{2}}, \\
& B^{(\mathrm{e})}\left(s^{j}, x^{i}\right)=2 \frac{y_{k} y_{l} n_{k} \bar{n}_{l}}{\bar{r}_{i j}^{4}}-\frac{n_{k} \bar{n}_{k}}{\bar{r}_{i j}^{2}},
\end{aligned}
$$

where $\bar{r}_{i j}=\left|s^{j}-x^{i}\right|, n_{k}$ is the $k$ th component of the outward normal vector at $s^{j} ; \bar{n}_{k}$ is the $k$ th component of the outward normal vector at $x^{i}$ and $y_{k}=x_{k}^{i}-s_{k}^{j}$. The chosen RBF is a kind of the two-point function.

It is noted that the double layer potentials have both singularity and hypersingularity at the origin, which lead to troublesome singular kernels and controversially auxiliary boundary in the conventional MFS. The off-set distance between the off-set (auxiliary) boundary $\left(B^{\prime}\right)$ and the real boundary $(B)$ defined by, $d$, as shown in Figs. 1(a) and (b) needs to be chosen deliberately. To overcome the abovementioned drawback, $s^{j}$ is distributed on the real boundary as shown in Figs. 1(c) and (d) by using the proposed regularization technique. The rationale for choosing double layer potential instead of the single layer potential as used in the proposed method for the form of RBFs is to take advantage of the desingularization of the subtracting and adding-back technique, so that no off-set distance is needed when evaluating the diagonal coefficients of influence matrices as explained in Section 3. The single layer potential will not be chosen as the form of RBFs, because Eqs. (20) and (21) in Section 3 are not satisfied. If the single layer potential is used, the desingularization of subtracting and adding-back technique will fail. 


\section{Derivation of diagonal coefficients of influence matrices for arbitrary domain}

When the collocation point $x^{i}$ approaches to the source point $s^{j}$, Eqs. (4) and (5) will become singular. Eqs. (4) and (5) for the interior problems need to be regularized by using special treatment of the desingularization of subtracting and adding-back technique $[13,17]$ as follows:

$$
\begin{aligned}
\phi\left(x^{i}\right) & =\sum_{j=1}^{N} A^{(\mathrm{i})}\left(s^{j}, x^{i}\right) \alpha^{j}-\sum_{j=1}^{N} A^{(\mathrm{e})}\left(s^{j}, x^{i}\right) \alpha^{i} \\
& =\sum_{j=1}^{i-1} A^{(\mathrm{i})}\left(s^{j}, x^{i}\right) \alpha^{j}+\sum_{j=i+1}^{N} A^{(\mathrm{i})}\left(s^{j}, x^{i}\right) \alpha^{j}+\left[\sum_{m=1}^{N} A^{(\mathrm{i})}\left(s^{m}, x^{i}\right)-A^{(\mathrm{i})}\left(s^{i}, x^{i}\right)\right] \alpha^{i}, \quad x^{i} \in B, \\
\psi\left(x^{i}\right) & =\sum_{j=1}^{N} B^{(\mathrm{i})}\left(s^{j}, x^{i}\right) \alpha^{j}-\sum_{j=1}^{N} B^{(\mathrm{e})}\left(s^{j}, x^{i}\right) \alpha^{i} \\
& =\sum_{j=1}^{i-1} B^{(\mathrm{i})}\left(s^{j}, x^{i}\right) \alpha^{j}+\sum_{j=i+1}^{N} B^{(\mathrm{i})}\left(s^{j}, x^{i}\right) \alpha^{j}-\left[\sum_{m=1}^{N} B^{(\mathrm{i})}\left(s^{m}, x^{i}\right)-B^{(\mathrm{i})}\left(s^{i}, x^{i}\right)\right] \alpha^{i}, \quad x^{i} \in B
\end{aligned}
$$

in which

$$
\begin{aligned}
& \sum_{j=1}^{N} A^{(\mathrm{e})}\left(s^{j}, x^{i}\right)=0, \quad x^{i} \in B, \\
& \sum_{j=1}^{N} B^{(\mathrm{e})}\left(s^{j}, x^{i}\right)=0, \quad x^{i} \in B .
\end{aligned}
$$

In Appendix A, the detail derivations of Eqs. (20) and (21) are given. The original singular terms of $A^{(\mathrm{i})}\left(s^{i}, x^{i}\right)$ and $B^{(\mathrm{i})}\left(s^{i}, x^{i}\right)$ in Eqs. (4) and (5) have been transformed into regular terms $\left[\sum_{m=1}^{N} A^{(\mathrm{i})}\right.$ $\left.\left(s^{m}, x^{i}\right)-A^{(\mathrm{i})}\left(s^{i}, x^{i}\right)\right]$ and $-\left[\sum_{m=1}^{N} B^{(\mathrm{i})}\left(s^{m}, x^{i}\right)-B^{(\mathrm{i})}\left(s^{i}, x^{i}\right)\right]$ in Eqs. (18) and (19), respectively. In which the terms of $\sum_{m=1}^{N} A^{(\mathrm{i})}\left(s^{m}, x^{i}\right)$ and $\sum_{m=1}^{N} B^{(\mathrm{i})}\left(s^{m}, x^{i}\right)$ are the adding-back terms and the terms of $A^{(\mathrm{i})}\left(s^{i}, x^{i}\right)$ and $B^{(i)}\left(s^{i}, x^{i}\right)$ are the subtracting terms in the two brackets for the special treatment technique. After using

\begin{tabular}{|c|c|c|c|c|}
\hline \multirow{2}{*}{$\begin{array}{l}\text { Kernel function } \\
\text { Eigenvalue } \lambda_{1}\end{array}$} & \multicolumn{2}{|l|}{$A\left(s^{j}, x^{i}\right)=-\frac{y_{k} n_{k}}{\bar{r}_{i j}^{2}}$} & \multicolumn{2}{|c|}{$B\left(s^{j}, x^{i}\right)=\frac{2 y_{k} y_{l} n_{k} \bar{n}_{l}}{\bar{r}_{i j}^{4}}-\frac{n_{k} \bar{n}_{k}}{\bar{r}_{i j}^{2}}$} \\
\hline & $\begin{array}{l}\text { Exterior } \\
v_{0}^{(\mathrm{e})}=0, v_{l}^{(\mathrm{e})}=\frac{N}{2 r}\end{array}$ & $\begin{array}{l}\text { Interior } \\
v_{0}^{(\mathrm{i})}=\frac{N}{r}, v_{l}^{(\mathrm{i})}=\frac{N}{2 r}\end{array}$ & $\begin{array}{l}\text { Exterior } \\
\delta_{0}^{(\mathrm{e})}=0, \delta_{l}^{(\mathrm{e})}=\frac{N l}{2 r^{2}}\end{array}$ & $\begin{array}{l}\text { Interior } \\
\delta_{0}^{(\mathrm{i})}=0, \delta_{l}^{(\mathrm{i})}=\frac{N l}{2 r^{2}}\end{array}$ \\
\hline \multirow{4}{*}{ Diagonal value } & \multicolumn{4}{|c|}{ Analytical solution: $\frac{1}{N} \sum_{m=0}^{N-1} \lambda_{m}=\frac{\text { Sum of diagonal terms }}{N}$ (circular domain only) } \\
\hline & $\frac{N-1}{2 r} \approx \frac{\pi}{\frac{2 \pi r}{N}}$ & $\frac{N+1}{2 r} \approx \frac{\pi}{\frac{2 \pi r}{N}}$ & $\frac{N(N-1)}{4 r^{2}} \approx \frac{\pi^{2}}{\left(\frac{2 \pi r}{N}\right)^{2}}$ & $\frac{N(N-1)}{4 r^{2}} \approx \frac{\pi^{2}}{\left(\frac{2 \pi r}{N}\right)^{2}}$ \\
\hline & \multicolumn{4}{|c|}{ Numerical solution (arbitrary domain) } \\
\hline & $\sum_{k=1}^{N} a_{i, k}-a_{i, i}$ & $\sum_{k=1}^{N} a_{i, k}-a_{i, i}$ & $-\left(\sum_{k=1}^{N} b_{i, k}-b_{i, i}\right)$ & $-\left(\sum_{k=1}^{N} b_{i, k}-b_{i, i}\right)$ \\
\hline
\end{tabular}
the desingularization of subtracting and adding-back technique [13,17], we are able to remove the singularity and hypersingularity of the kernel functions. Therefore, the diagonal coefficients for the interior problems can be extracted out as:

Table 1

The properties of the influence matrices for the Laplace equation

Where $\bar{r}_{i j}=\left|x^{i}-s^{j}\right|, y_{k}=x_{k}^{i}-s_{k}^{j}, \bar{n}_{k}$ denotes the $k$ th components of outward normal vector on $x^{i}$, respectively. 

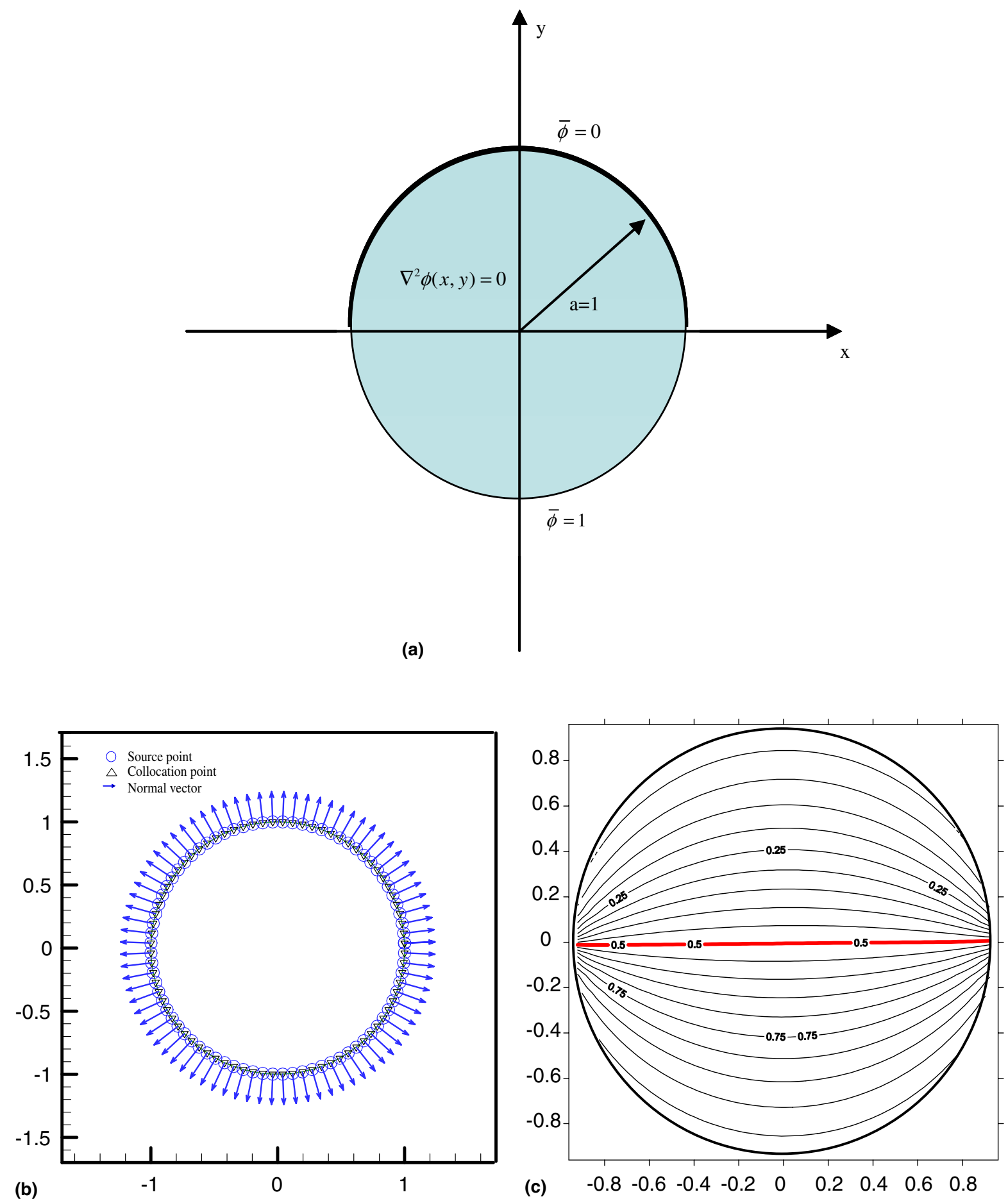

Fig. 2. Problem sketch and the nodes distribution (60 nodes) in the case 1.1: (a) problem sketch, (b) nodes distribution, (c) exact solution. 

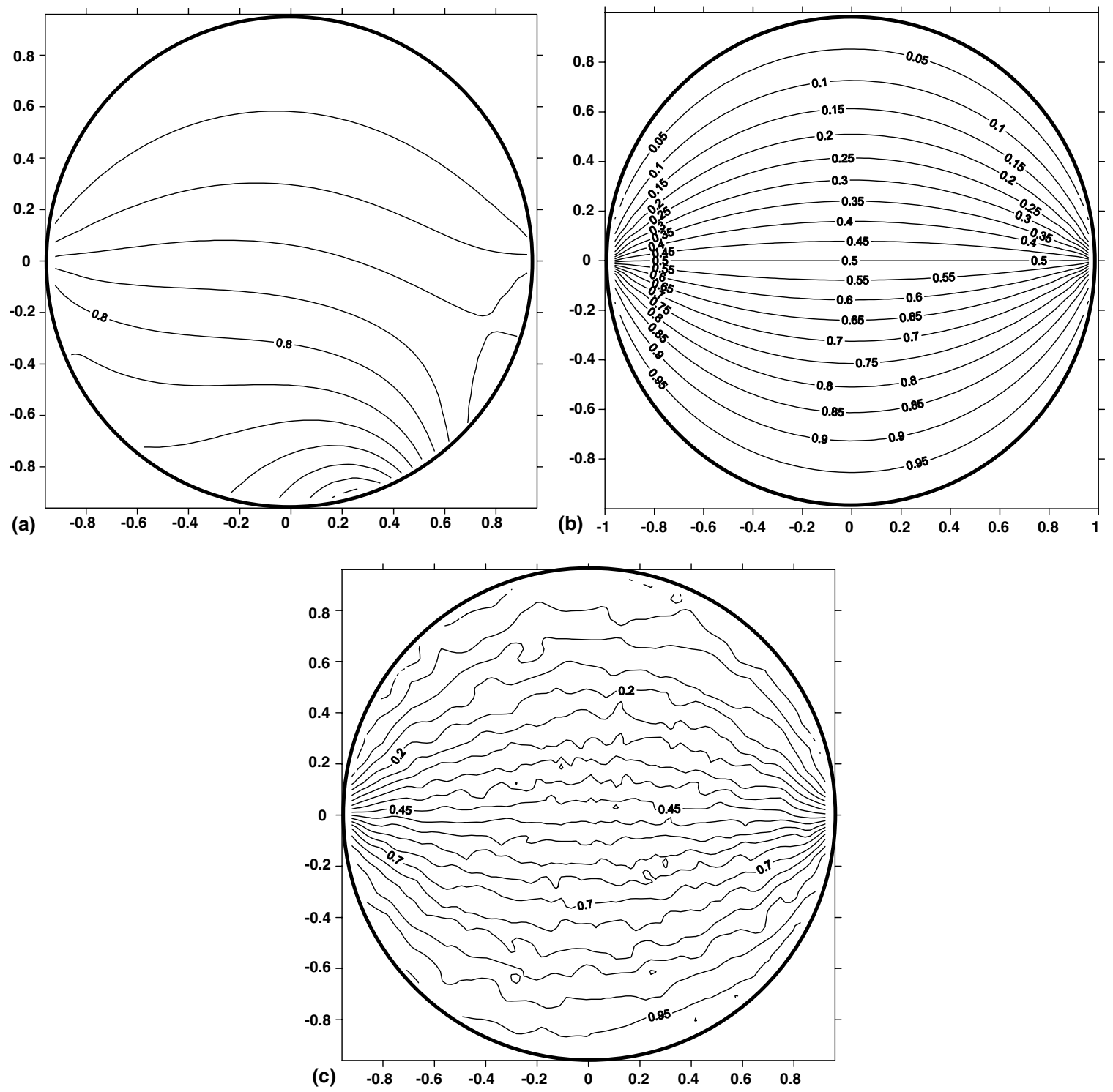

Fig. 3. The field solutions by using the conventional MFS (60 nodes) for the case 1.1: (a) $d=0.01$, (b) $d=0.5$, (c) $d=1.0$.

$$
\left\{\phi^{i}\right\}=\left[\begin{array}{cccc}
\sum_{m=1}^{N} a_{1, m}-a_{1,1} & a_{1,2} & \cdots & a_{1, N} \\
a_{2,1} & \sum_{m=1}^{N} a_{2, m}-a_{2,2} & \cdots & a_{2, N} \\
\vdots & \vdots & \ddots & \vdots \\
a_{N, 1} & a_{N, 2} & \cdots & \sum_{m=1}^{N} a_{N, m}-a_{N, N}
\end{array}\right]\left\{\alpha^{j}\right\}
$$



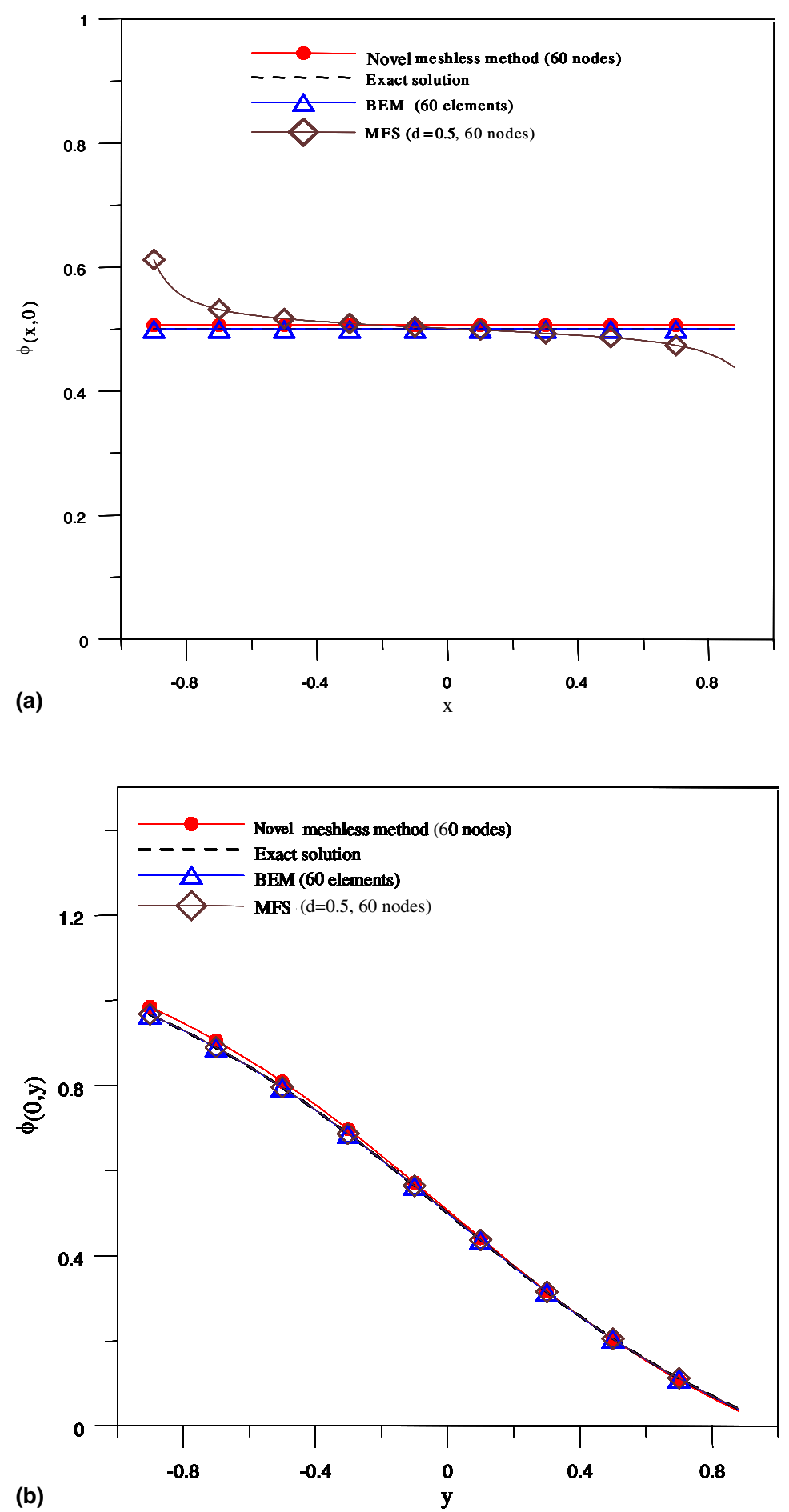

Fig. 4. The field solutions by using the proposed method, BEM and conventional MFS for the case $1.1 ;(\mathrm{a}) \phi(x, 0),(\mathrm{b}) \phi(0, y)$. 


$$
\left\{\psi^{i}\right\}=\left[\begin{array}{cccc}
-\left(\sum_{m=1}^{N} b_{1, m}-b_{1,1}\right) & b_{1,2} & \cdots & b_{1, N} \\
b_{2,1} & -\left(\sum_{m=1}^{N} b_{2, m}-b_{2,2}\right) & \cdots & b_{2, N} \\
\vdots & \vdots & \ddots & \vdots \\
b_{N, 1} & b_{N, 2} & \cdots & -\left(\sum_{m=1}^{N} b_{N, m}-b_{N, N}\right)
\end{array}\right]\left\{\alpha^{j}\right\} .
$$

In a similar way, the desingularization of subtracting and adding-back technique was applied to the exterior problems, we then have

$$
\begin{aligned}
& \phi\left(x^{i}\right)=\sum_{j=1}^{N} A^{(\mathrm{e})}\left(s^{j}, x^{i}\right) \alpha^{j}-\sum_{j=1}^{N} A^{(\mathrm{e})}\left(s^{j}, x^{i}\right) \alpha^{i}, \\
& \psi\left(x^{i}\right)=\sum_{j=1}^{N} B^{(\mathrm{e})}\left(s^{j}, x^{i}\right) \alpha^{j}-\sum_{j=1}^{N} B^{(\mathrm{e})}\left(s^{j}, x^{i}\right) \alpha^{i} .
\end{aligned}
$$

After using Eqs. (14) and (15), the diagonal coefficients for the exterior problems can be extracted out as:

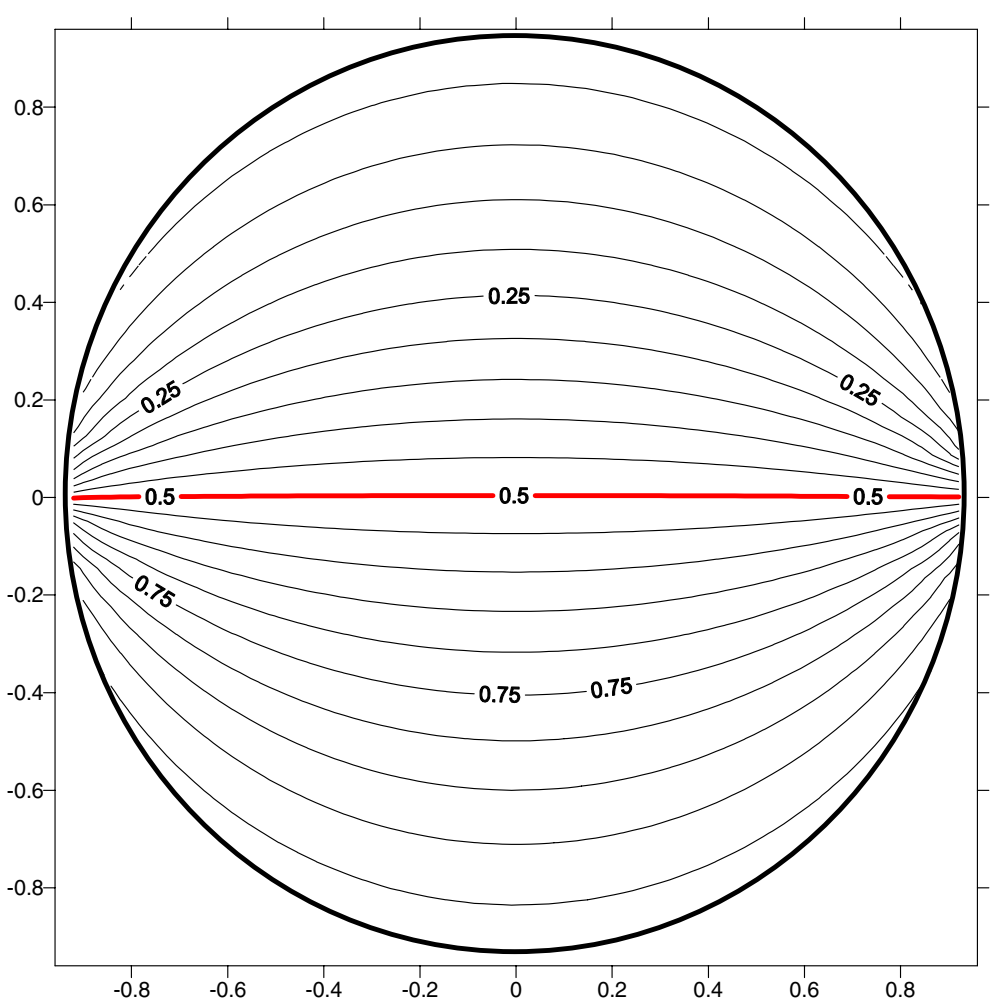

Fig. 5. The field solutions for the case 1.1 by using the novel meshless method ( 60 nodes). 

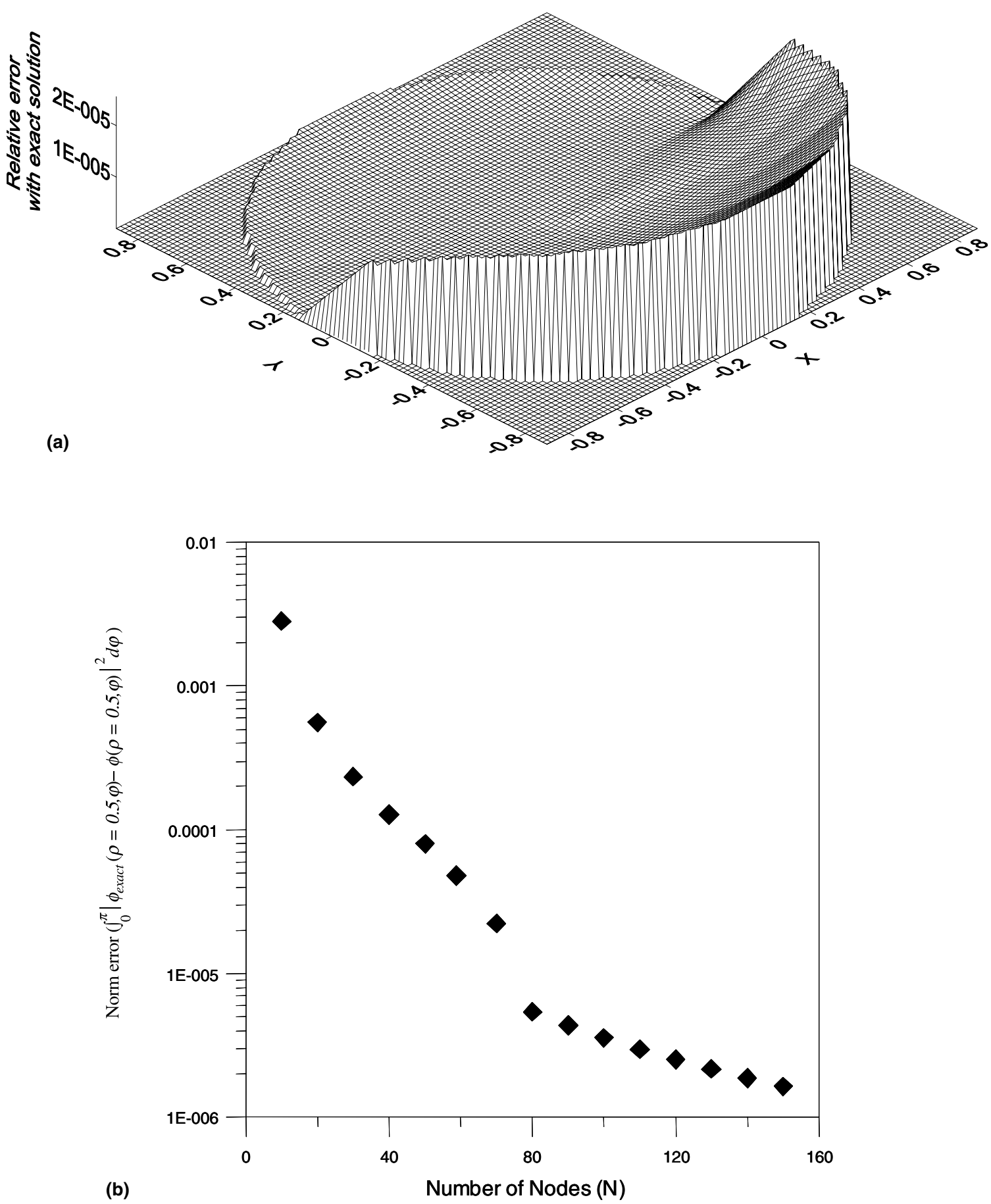

Fig. 6. The error analyses for the case 1.1: (a) relative error with exact solution for entire domain (60 source nodes), (b) norm error (at radius $=0.5)$ versus the numbers $(N)$ of nodes. 
$\left\{\phi^{i}\right\}=\left[\begin{array}{cccc}\sum_{m=1}^{N} a_{1, m}-a_{1,1} & -a_{1,2} & \cdots & -a_{1, N} \\ -a_{2,1} & \sum_{m=1}^{N} a_{2, m}-a_{2,2} & \cdots & -a_{2, N} \\ \vdots & \vdots & \ddots & \vdots \\ -a_{N, 1} & -a_{N, 2} & \cdots & \sum_{m=1}^{N} a_{N, m}-a_{N, N}\end{array}\right]\left\{\alpha^{j}\right\}$,

(a)
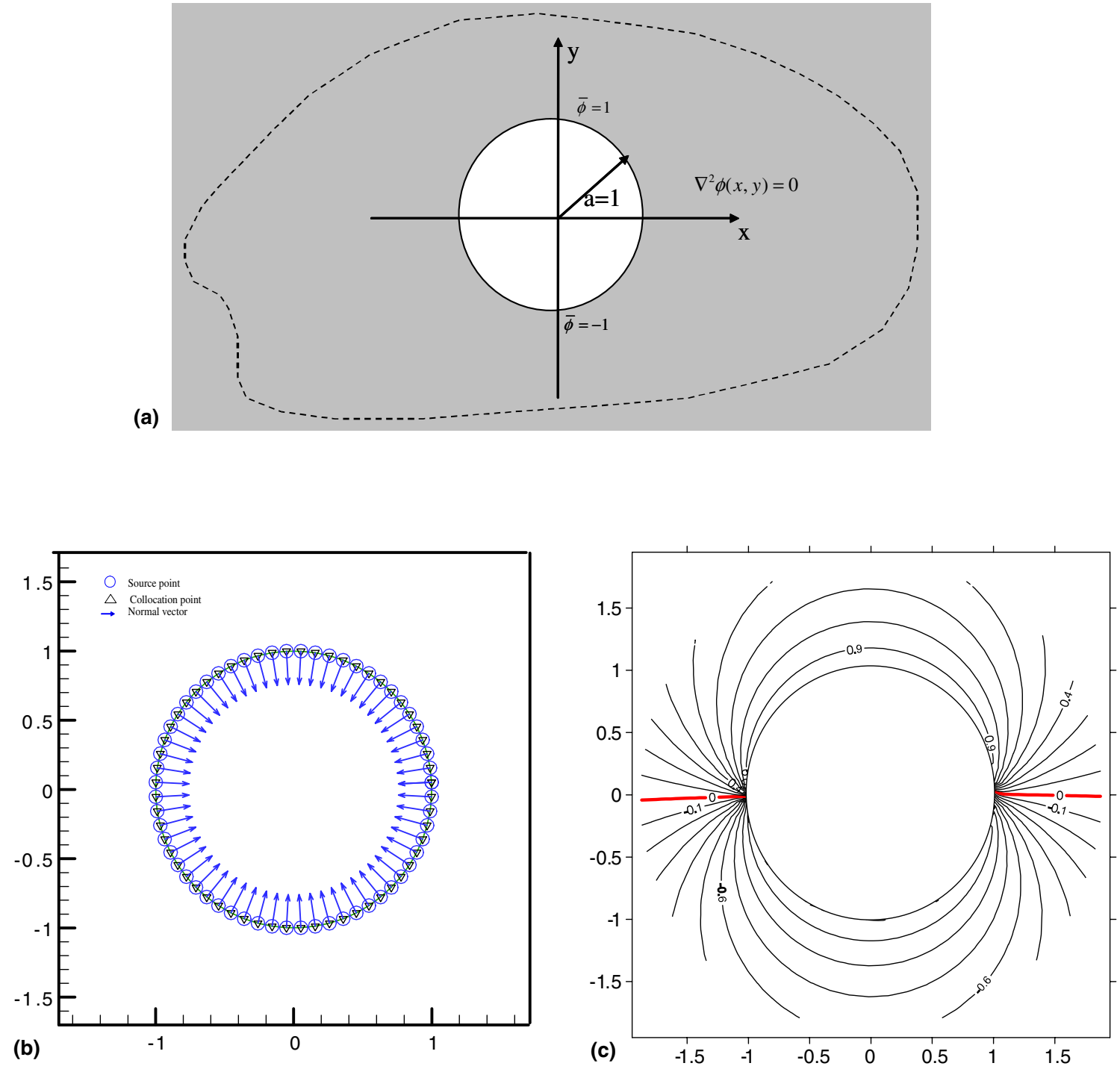

Fig. 7. Problem sketch and the nodes distribution (60 nodes) in the case 1.2: (a) problem sketch, (b) nodes distribution, (c) exact solution. 
(a)
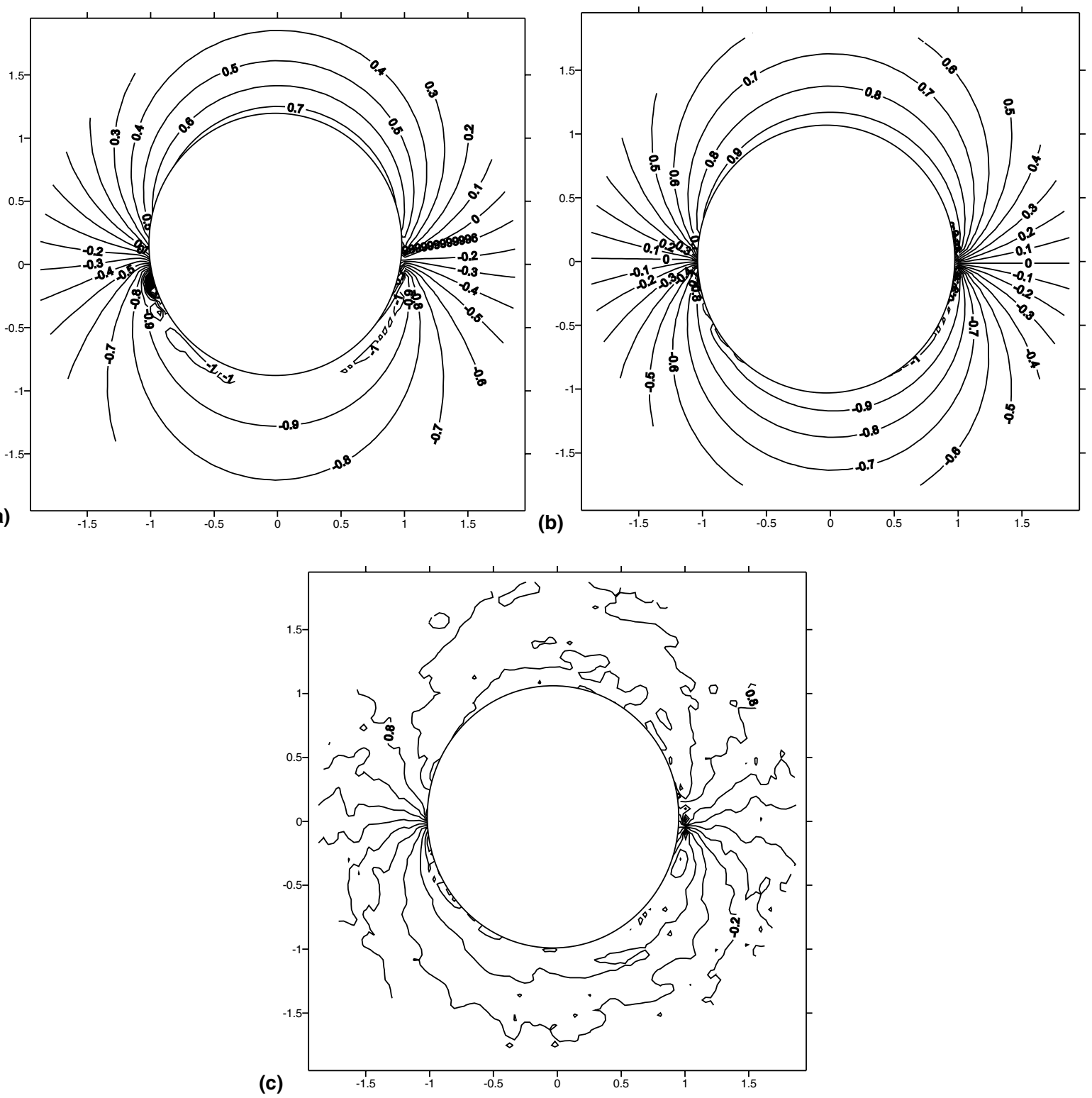

Fig. 8. The field solutions by using the conventional MFS (60 nodes) for the case 1.2: (a) $d=0.001$, (b) $d=0.2$, (c) $d=0.5$.

$$
\left\{\psi^{i}\right\}=\left[\begin{array}{cccc}
-\left(\sum_{m=1}^{N} b_{1, m}-b_{1,1}\right) & b_{1,2} & \cdots & b_{1, N} \\
b_{2,1} & -\left(\sum_{m=1}^{N} b_{2, m}-b_{2,2}\right) & \cdots & b_{2, N} \\
\vdots & \vdots & \ddots & \vdots \\
b_{N, 1} & b_{N, 2} & \cdots & -\left(\sum_{m=1}^{N} b_{N, m}-b_{N, N}\right)
\end{array}\right]\left\{\alpha^{j}\right\} .
$$




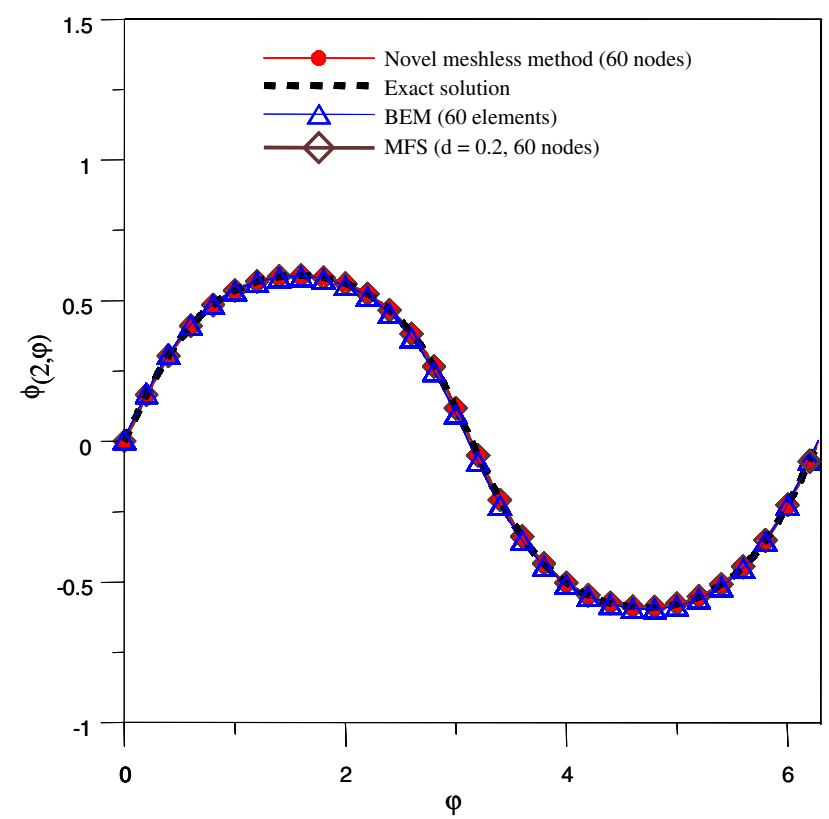

Fig. 9. The field solutions, $\phi(2, \varphi)$, by using the proposed method, BEM and conventional MFS for the case 1.2 .

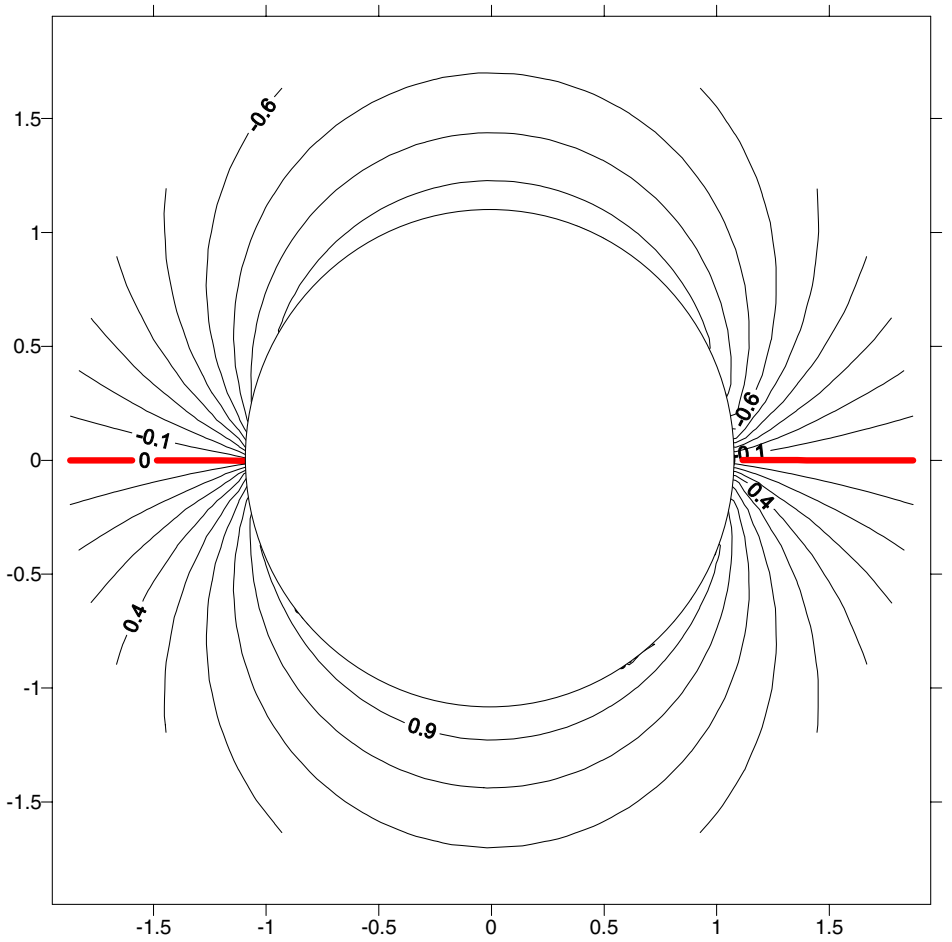

Fig. 10. The field solution for the case 1.2 by using the novel meshless method (60 nodes). 
The diagonal terms of the two influence matrices for both interior and exterior problems can also be derived analytically for a circular domain as shown in Appendix B. Table 1 shows the properties of the influence matrices for both circular and arbitrary domains.

\section{Numerical results}

In order to show the accuracy and validity of the proposed method, the potential problems with circular, square and arbitrary domains subject to the Dirichlet, Neumann, and mixed-type problems with continuous and discontinuous BCs are considered. The results will be compared to the solutions obtained by using the conventional MFS, BEM and exact solutions.

\subsection{Example 1: Circular domain cases (cases 1.1 and 1.2)}

In cases 1.1 and 1.2, the interior and exterior Dirichlet problems with discontinuous BCs are given. The interior Dirichlet problem is considered in case 1.1. Case 1.2 is the exterior Dirichlet problem. Both figures in the following two cases are the results with 60 source nodes.
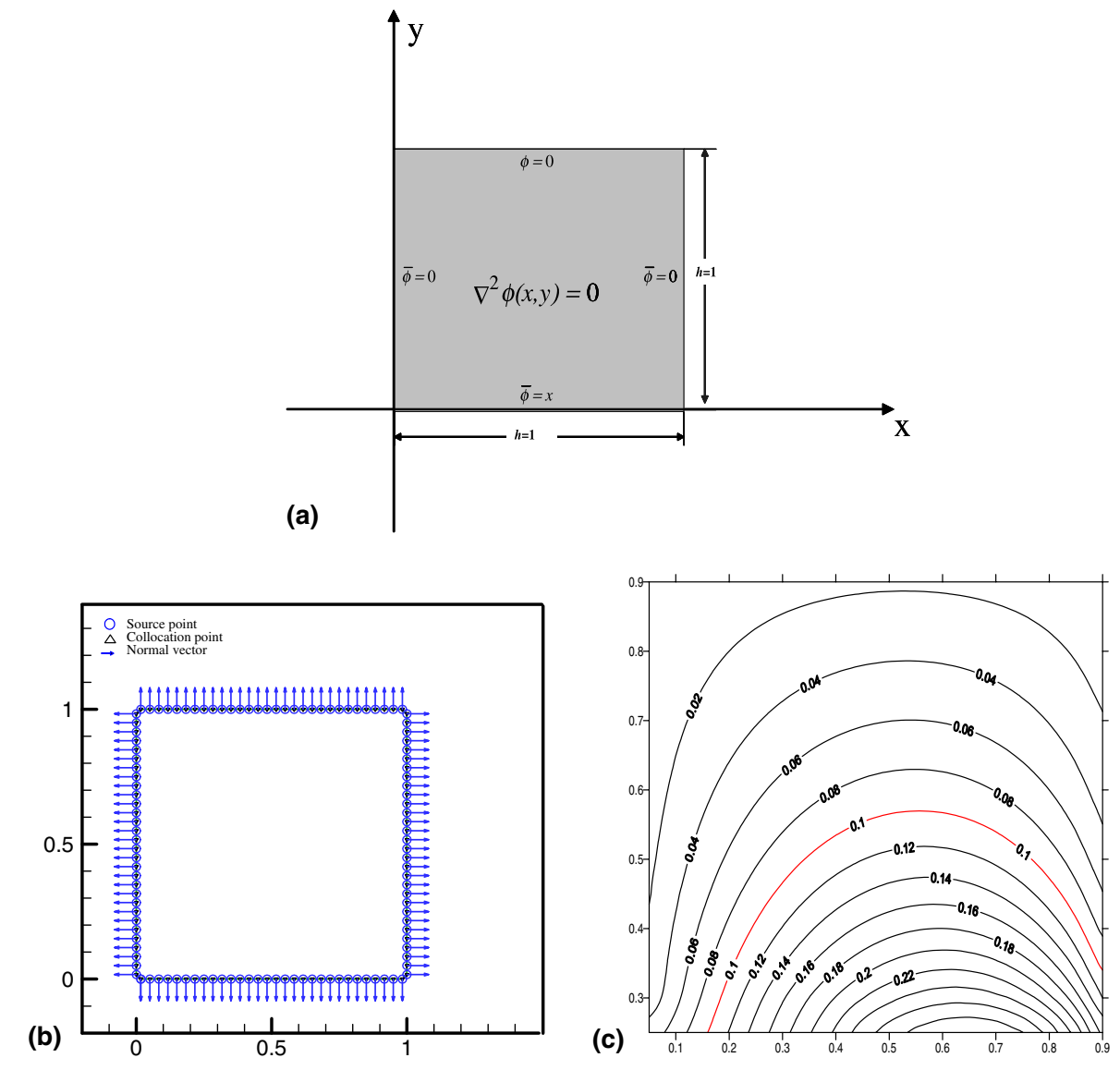

Fig. 11. Problem sketch and the nodes distribution (120 nodes) in the case 2.1: (a) problem sketch, (b) nodes distribution, (c) exact solution. 


\subsubsection{Case 1.1: interior Dirichlet problem}

Problem sketch and the nodes distribution employing the proposed method are depicted in Figs. 2(a) and (b), respectively. The problem is subject to Dirichlet discontinuous BC as follows:

$$
\bar{\phi}(1, \varphi)= \begin{cases}0, & 0<\varphi<\pi \\ 1, & \pi<\varphi<2 \pi\end{cases}
$$
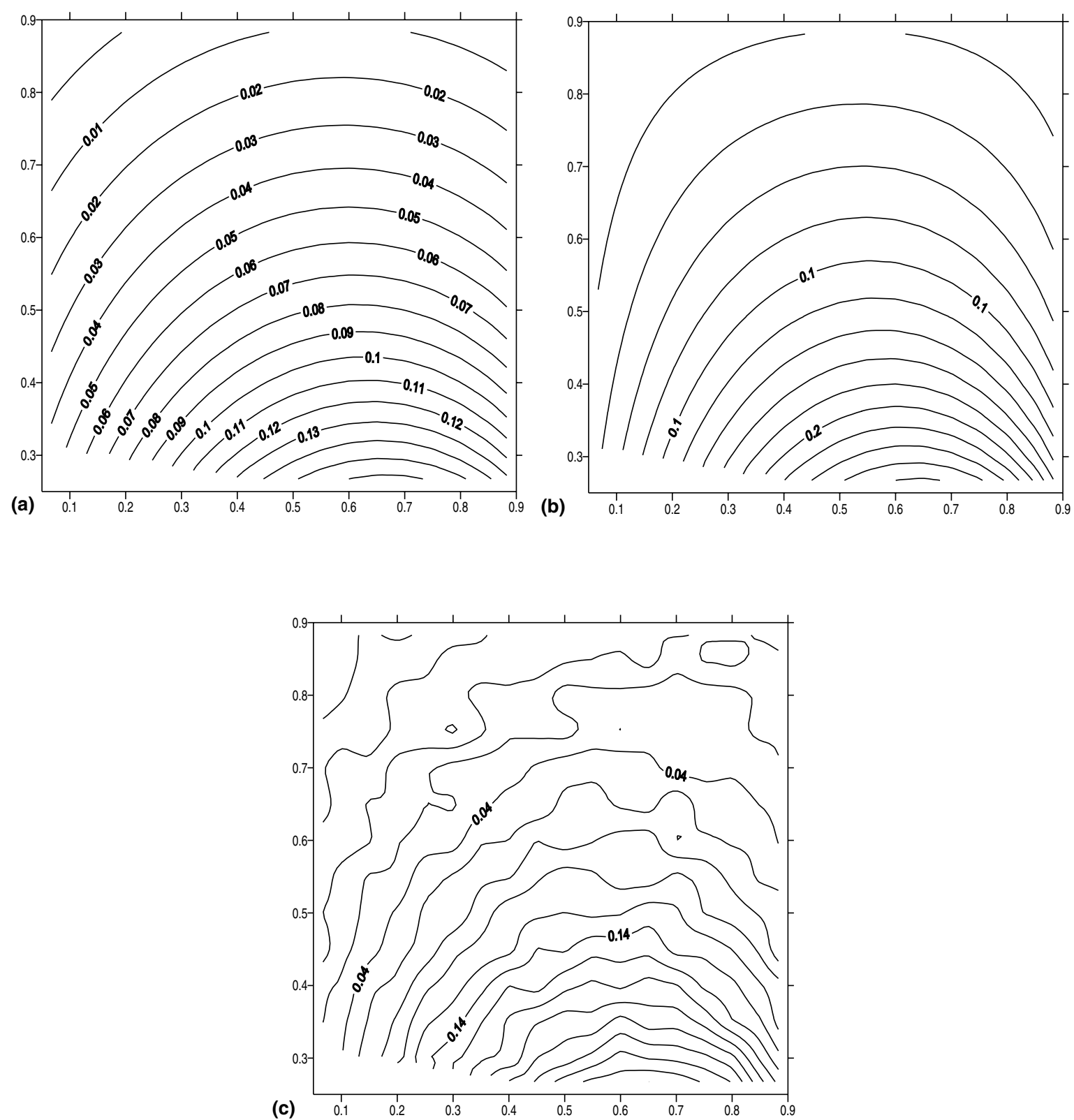

Fig. 12. The field solutions by using the conventional MFS (120 nodes) for the case 2.1 : (a) $d=0.1$, (b) $d=0.5$, (c) $d=1.0$. 
In this case, an analytical solution is found as follows:

$$
\phi(x, y)=\frac{1}{\pi} \arctan \left(\frac{1-x^{2}-y^{2}}{2 y}\right) .
$$

The exact field solution is plotted in Fig. 2(c). We obtain the results of the field potential solutions by using the conventional MFS for distributing source points (60 nodes) on the fictitious boundary with different
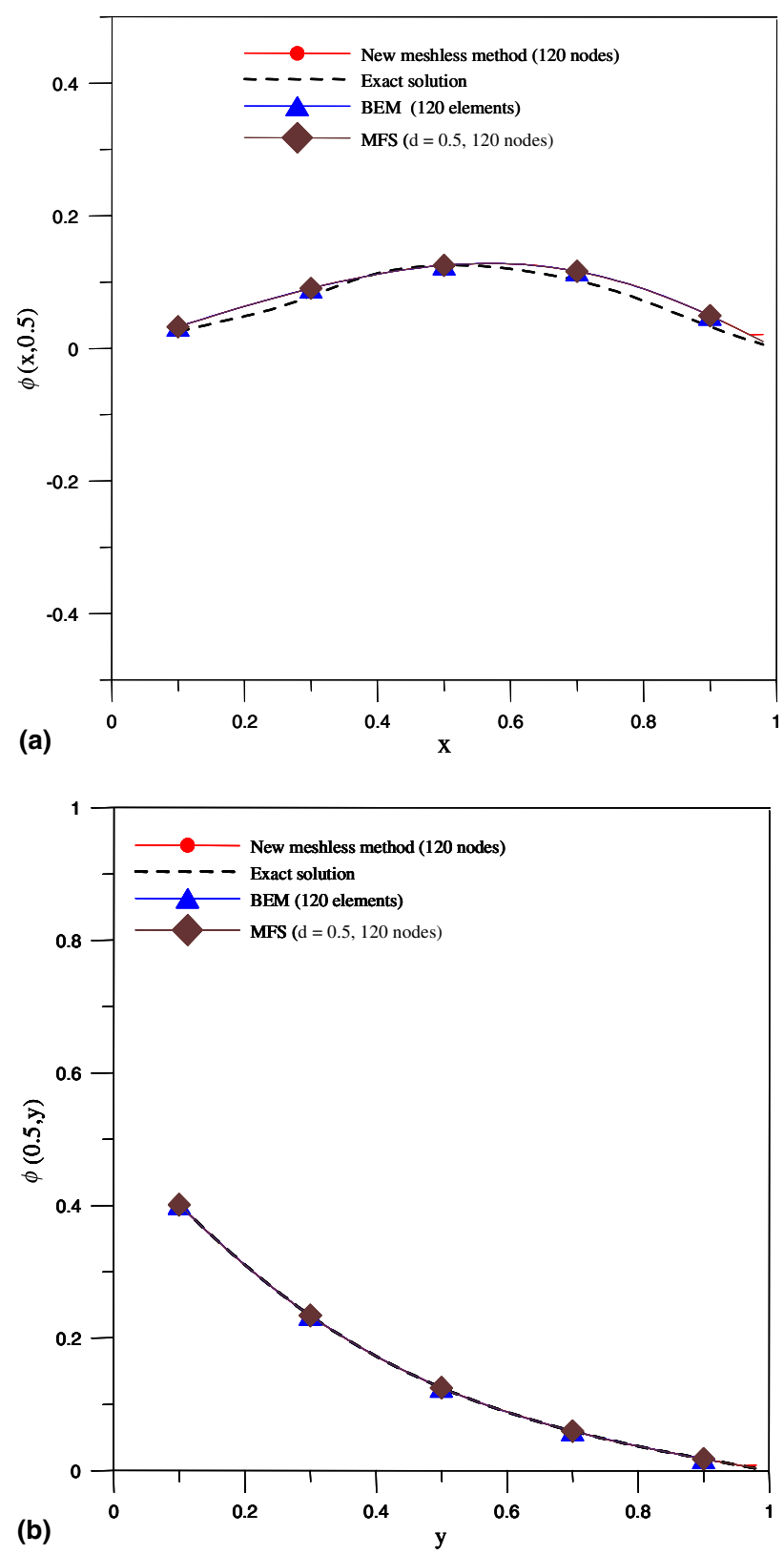

Fig. 13. The field solutions by using the proposed method, BEM and conventional MFS by adding a rigid body term for the case 2.1: (a) $\phi(x, 0.5)$, (b) $\phi(0.5, y)$. 
off-set distances as depicted in Figs. 3(a)-(c). It is obvious that the relative errors of the conventional MFS comparing with the exact solution in Fig. 2(c) for $d=0.01$ and $d=1.0$ are larger than $d=0.5$, where $d$ is the distance between the off-set (auxiliary) boundary $\left(B^{\prime}\right)$ and the real boundary $(B)$. This illustrates the drawback that the location of source is dubious by using the conventional MFS. The comparisons of results by using the proposed novel method, the conventional MFS $(d=0.5)$, the BEM, and the analytical solution are displayed in Fig. 4(a) for $\phi(x, 0)$ and Fig. 4(b) for $\phi(0, y)$, respectively. The field solution of the present method is plotted in Fig. 5. The results match the exact solutions very well by using the present meshless method. To see the sensitivity analysis of the boundary layer effect, the relative error with exact solution in the interested domain field with 800 inner points is given in Fig. 6(a). Fig. 6(b) shows the norm error of the numerical results plotted versus number of nodes and displays the changes of norm error at radius $=0.5$ with the increase of source nodes $N$. The norm error is defined as $\int_{0}^{2 \pi} \mid \phi_{\text {exact }}(\rho=0.5, \varphi)-$ $\left.\phi(\rho=0.5, \varphi)\right|^{2} \mathrm{~d} \varphi$ in Fig. 6(b).

\subsubsection{Case 1.2: exterior Dirichlet problem}

In this case, we investigate a circular domain with the Dirichlet discontinuous BC given as follows:

$$
\bar{\phi}(1, \varphi)= \begin{cases}1, & 0<\varphi<\pi, \\ -1, & \pi<\varphi<2 \pi .\end{cases}
$$

Problem sketch and the nodes distribution using the proposed method are depicted in Figs. 7(a) and (b), respectively. In this case, an analytical solution is available as following:

$$
\phi(x, y)=\frac{2}{\pi} \arctan \left(\frac{2 y}{x^{2}+y^{2}-1}\right) .
$$

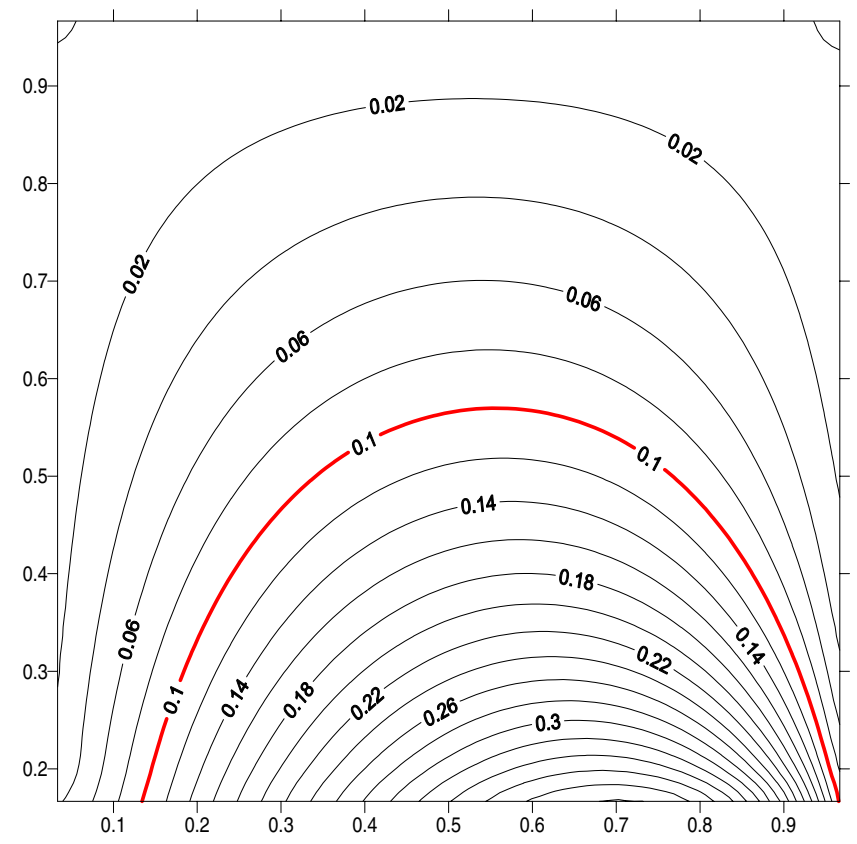

Fig. 14. The field solution of the case 2.1 by using the novel meshless method (120 nodes). 
The exact field solution is plotted in Fig. 7(c). We obtain the results of the field potential solutions by using the conventional MFS (60 nodes) for different off-set distances to boundary as shown in Figs. $8(\mathrm{a})-$ (c). The relative errors of the conventional MFS are larger for $d=0.001$ and $d=0.5$ than $d=0.2$. This clearly illustrates the drawback of the well-known ill-posed influence matrices by using the conventional MFS. The field results, $\phi(2, \varphi)$ by using the present novel method, the conventional MFS $(d=0.2)$, the BEM and the analytical solutions are plotted in Fig. 9. In Fig. 10 the field solution of the proposed technique is plotted. The present numerical results are very close to the exact solutions by using the proposed novel method.

\subsection{Example 2: square domain cases (cases 2.1 and 2.2)}

In cases 2.1 and 2.2, the interior Dirichlet and mixed-type problems are given for the square domain, respectively. The two problems considered here are all with discontinuous BCs.
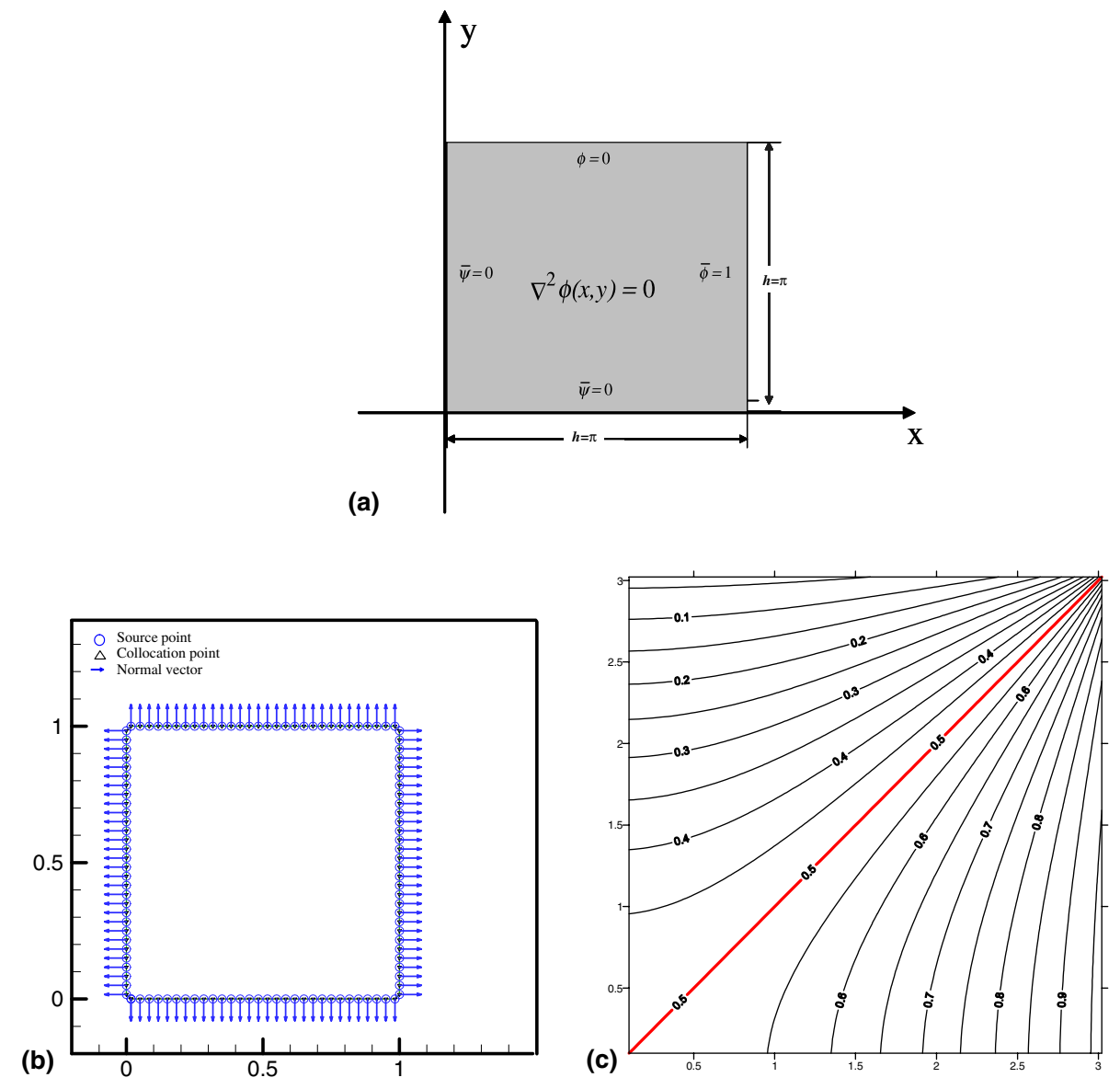

Fig. 15. Problem sketch and the nodes distribution (120 nodes) in the case 2.2: (a) problem sketch, (b) nodes distribution, (c) exact solution. 
4.2.1. Case 2.1: interior Dirichlet problem (discontinuous $B C$ )

A square domain $(1 \times 1)$ subject to the Dirichlet $\mathrm{BC}$ is considered as

$$
\phi(x, 0)=x, \quad \phi(x, 1)=\phi(0, y)=\phi(1, y)=0 .
$$

Problem sketch and the nodes distribution using the proposed method are depicted in Figs. 11(a) and (b), respectively. An analytical solution is available as follows:
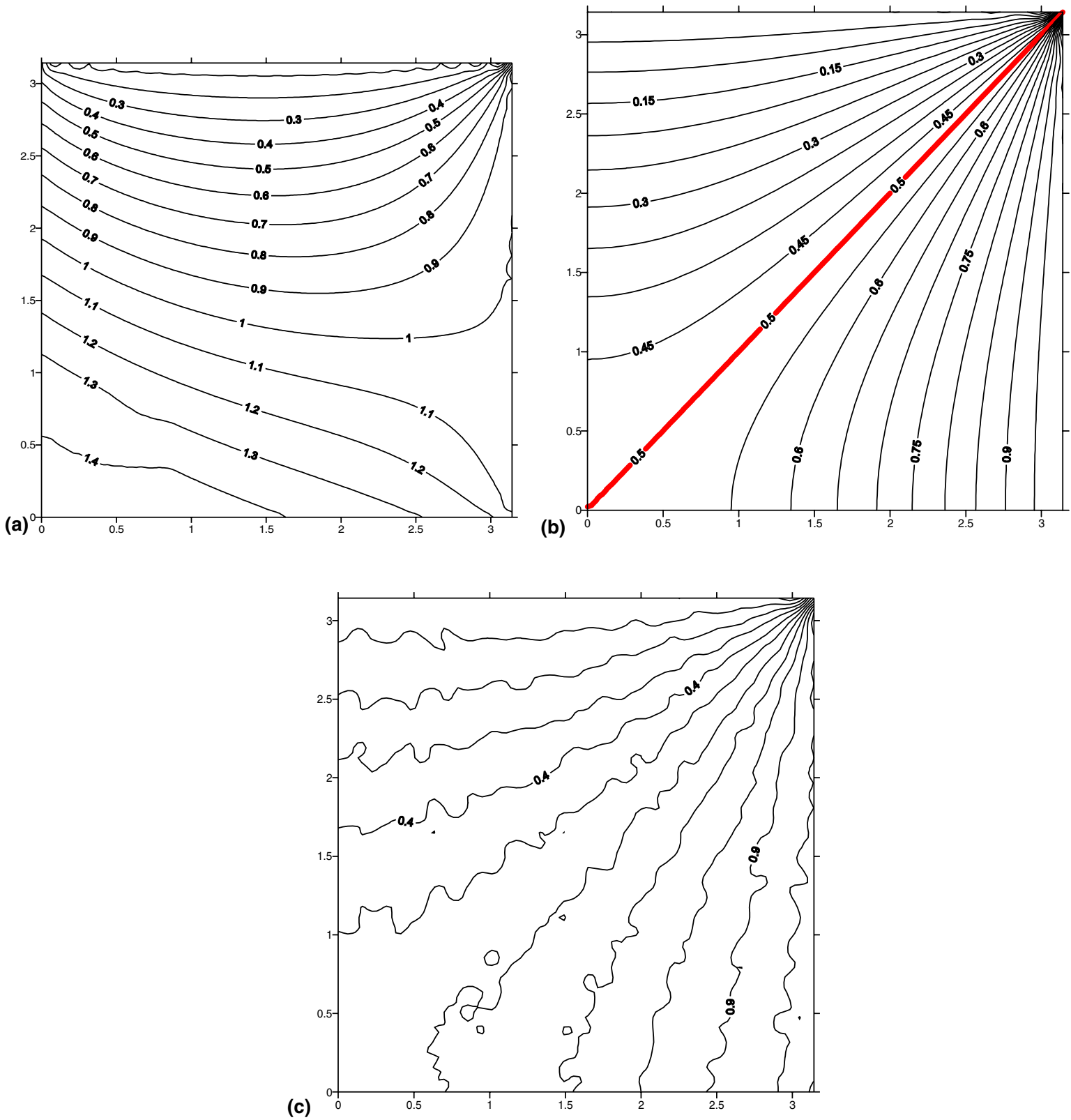

Fig. 16. The field solutions by using the conventional MFS (120 nodes) for the case 2.2: (a) $d=0.01$, (b) $d=0.5$, (c) $d=1.0$. 

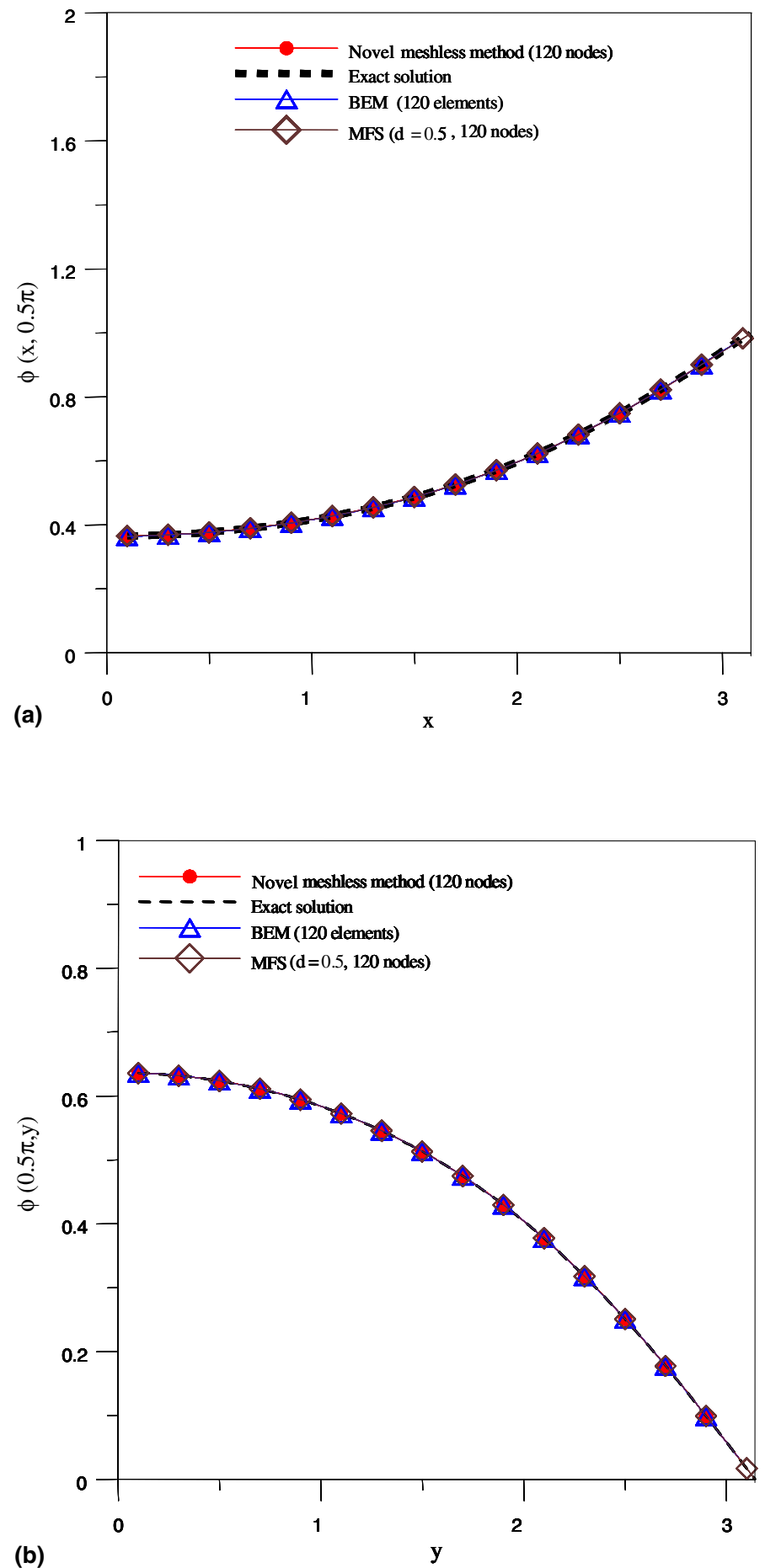

Fig. 17. The field solutions by using the proposed method, BEM and conventional MFS by adding a rigid body term for the case 2.2: (a) $\phi(x, 0.5 \pi)$, (b) $\phi(0.5 \pi, y)$. 


$$
\phi(x, y)=\sum_{n=1}^{\infty} C_{n} \sinh (n \pi(1-y)) \sin (n \pi x),
$$

where

$$
C_{n}=\frac{2(-1)^{n+1}}{(n \pi) \sinh (n \pi)} .
$$

The exact field solution is plotted in Fig. 11(c). After distributing 120 nodes, we obtain the results by using the conventional MFS for different off-set distances to boundary $(d)$ as depicted in Fig. 12, where $d$ is the off-set distance between the off-set (auxiliary) boundary $\left(B^{\prime}\right)$ and the real boundary $(B)$. It is obvious that the relative errors of the conventional MFS comparing with the exact solution in Fig. 11(c) for $d=0.1$ and $d=1$ are larger than $d=0.5$. This illustrates the important fact that the location of source is vital to the accuracy of the solution by using the conventional MFS. In such a situation, the conventional MFS does not yield reliable and consistent solutions. The field solutions of $\phi(x, 0.5)$ and $\phi$ $(0.5, y)$ by employing the proposed novel method, the conventional MFS $(d=0.5)$, the BEM and the analytical results are plotted in Figs. 13(a) and (b), respectively. The present method predicts the accurate solutions after comparing with the analytical solutions as shown in Fig. 13. Good match is observed from the comparison of the two solutions. Thus the selection of the off-set distances in the conventional MFS is avoided by adopting the present study. The field solution by using the proposed method is plotted in Fig. 14.

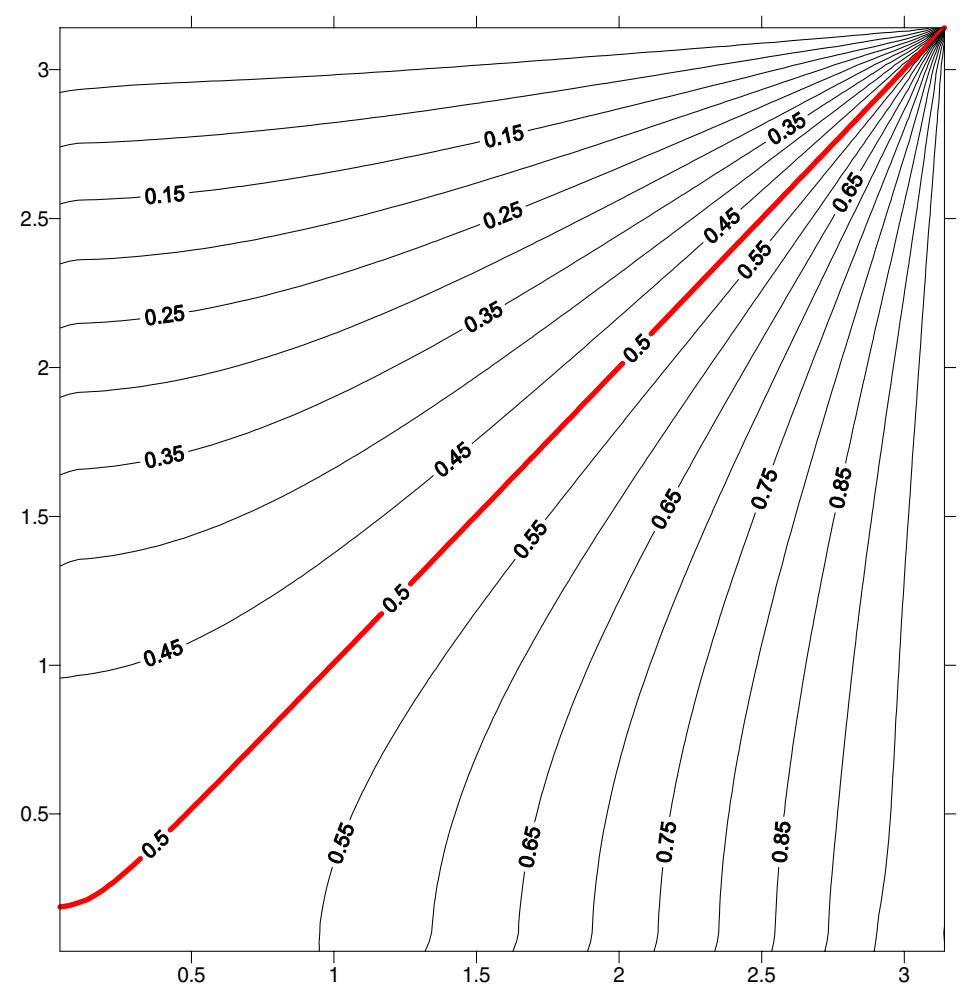

Fig. 18. The field solution for the case 2.2 by using the novel meshless method (120 nodes). 


\subsubsection{Case 2.2: interior mixed-type problem (discontinuous BC)}

A square domain $(\pi \times \pi)$ subject to the mixed-type $\mathrm{BC}$ is considered as

$$
\phi(\pi, y)=1, \quad \psi(x, 0)=\phi(x, \pi)=\psi(0, y)=0 .
$$

Problem sketch and the nodes distribution using the proposed method are depicted in Figs. 15(a) and (b), respectively. An analytical solution is available as follows:

$$
\phi(x, y)=\sum_{n=1}^{\infty} D_{n} \cosh \left(\frac{(2 n-1) x}{2}\right) \cos \left(\frac{(2 n-1) y}{2}\right),
$$

where

$$
D_{n}=\frac{4(-1)^{n+1}}{(2 n-1) \pi \cosh \left(\frac{(2 n-1) \pi}{2}\right)} .
$$

The field solution of the exact solution is plotted in Fig. 15(c). By collocating 120 nodes, we derive the results by using the conventional MFS for different values of $d$ as obtained in Fig. 16. It is obvious that the results of the conventional MFS for $d=0.01$ and $d=1.0$ are larger than $d=0.5$ after comparing with the exact solution in Fig. 15(c). The results of $\phi(x, 0.5 \pi)$ and $\phi(0.5 \pi, y)$ by using the proposed novel meshless method, the conventional MFS $(d=0.5)$, the BEM and the analytical solutions are plotted

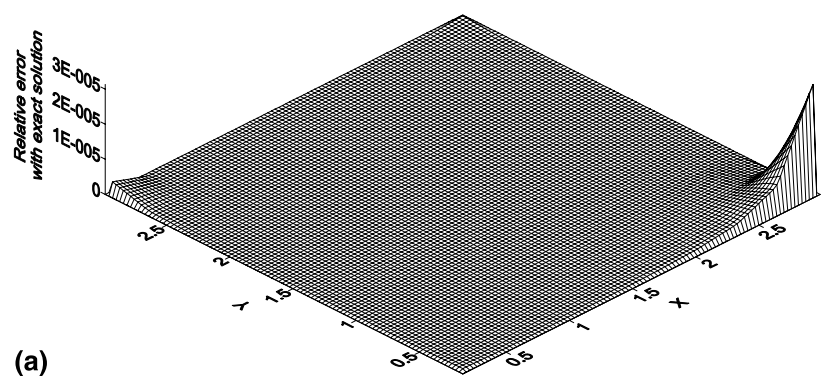

(a)

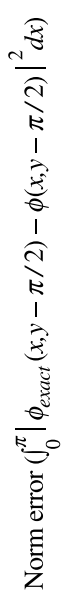

(b)

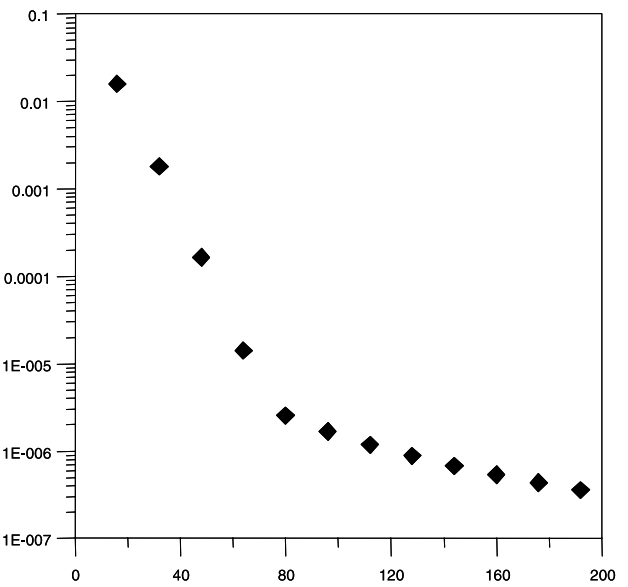

Number of nodes $(\mathrm{N})$ (c)

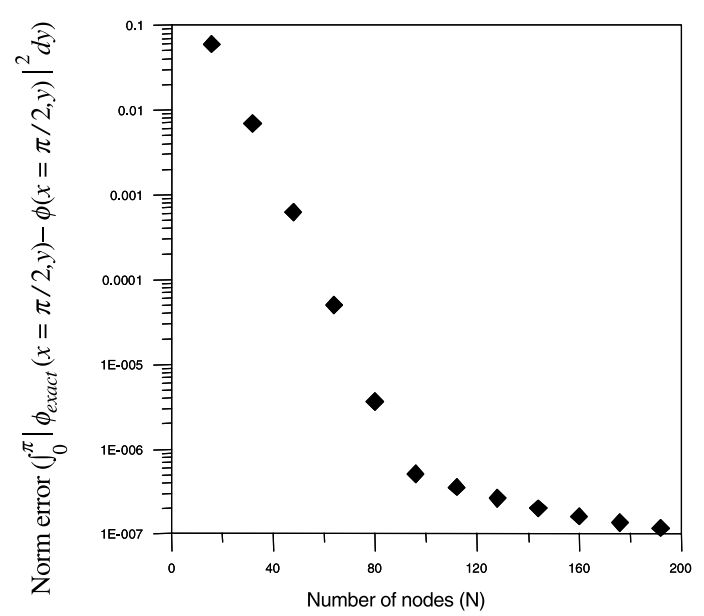

Fig. 19. The error analyses for the case 2.2: (a) relative error with exact solution for entire domain (120 source nodes), (b) norm error at line $\left(x, \frac{\pi}{2}\right)$, (c) norm error at line $\left(\frac{\pi}{2}, y\right)$. 


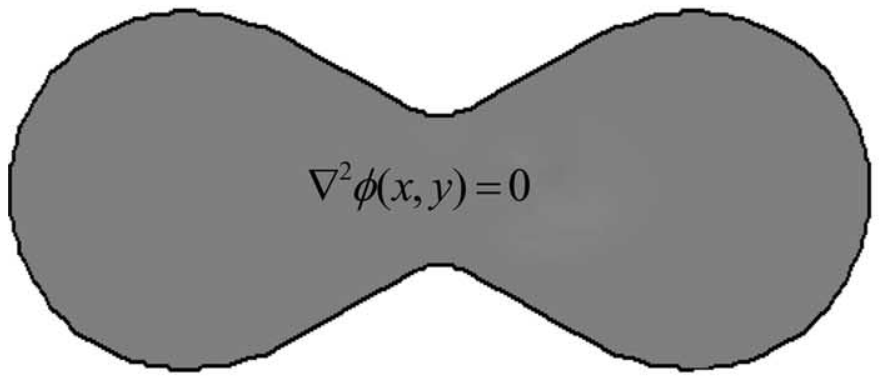

(a)

$$
\phi(x, y)=e^{x} \cos y,(x, y) \in \mathbf{B}
$$
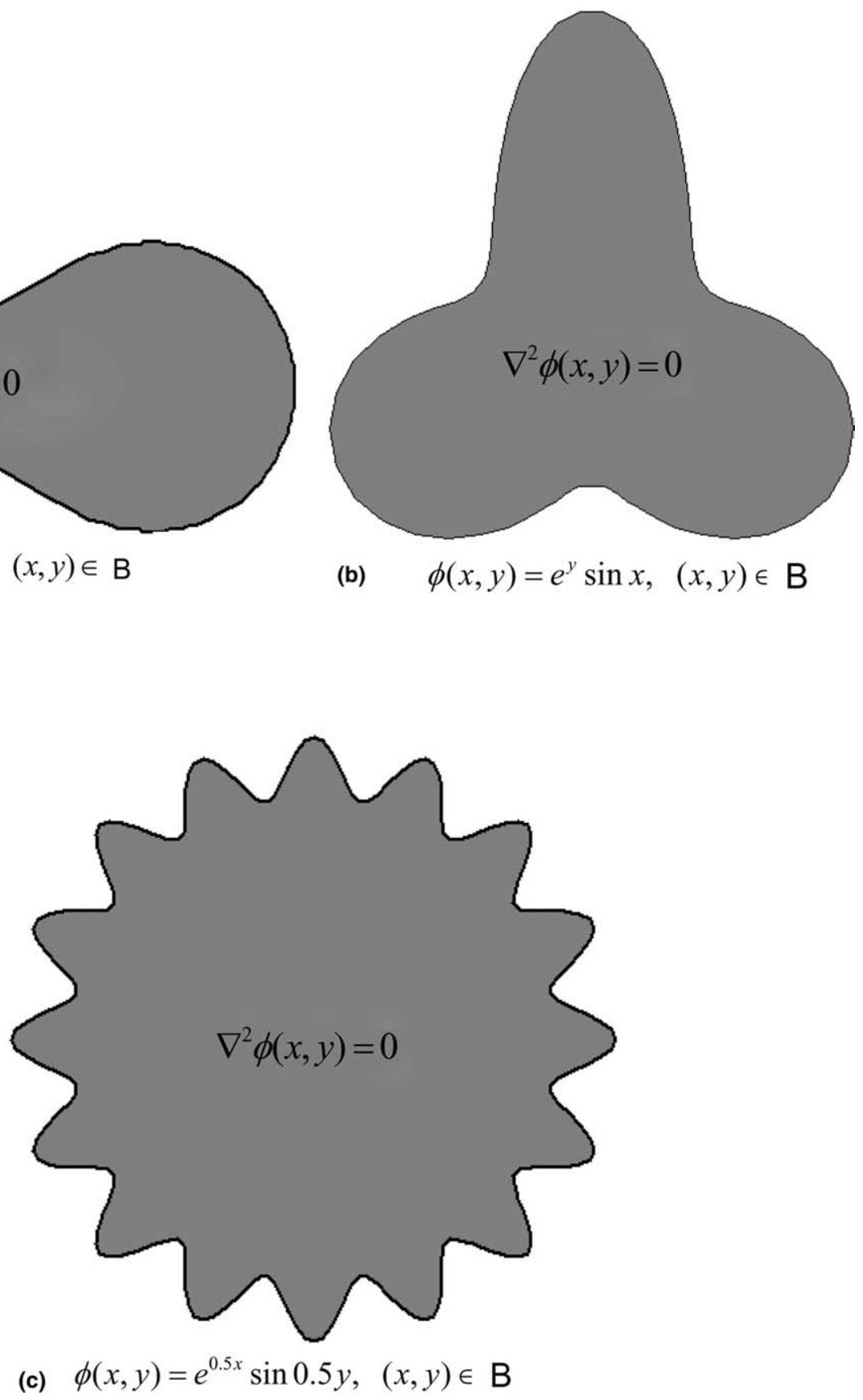

Fig. 20. Problems sketches in the case 3: (a) case 3.1, (b) case 3.2, (c) case 3.3.

in Figs. 17(a) and (b), respectively. The field solution by using the proposed method is plotted in Fig. 18. To investigate the error analysis, we plot Figs. 19(a)-(c). In Fig. 19(a), the relative error with exact solution in the interested domain with 400 inner points is plotted. Meanwhile Figs. 19(b) and (c) display 
the norm error along line $y=\pi / 2$ and $x=\pi / 2$ versus the number $N$ of boundary nodes. The norm errors are defined as $\int_{0}^{\pi}\left|\phi_{\text {exact }}(x, y=\pi / 2)-\phi(x, y=\pi / 2)\right|^{2} \mathrm{~d} x$ in Fig. 19(b) and $\int_{0}^{\pi} \mid \phi_{\text {exact }}(x=\pi / 2, y)-$ $\left.\phi(x=\pi / 2, y)\right|^{2} \mathrm{~d} y$ in Fig. 19(b), respectively. The boundary layer effect is observed in Fig. 6(a) for case 1.1 and Fig. 19(a) for case 2.2. When the observation points are calculated, in the proximity of the interior points to the boundary owing to the singularities of the double layer kernels, the precision would deteriorate quickly. In order to reduce the boundary layer effect to some extent, the remedy may be to refine the local source points. This has been verified from Fig. 6(b) for case 1.1 and Figs. 19(b) and (c) for case 2.2. As the node points are refined the boundary layer effects become less obvious.
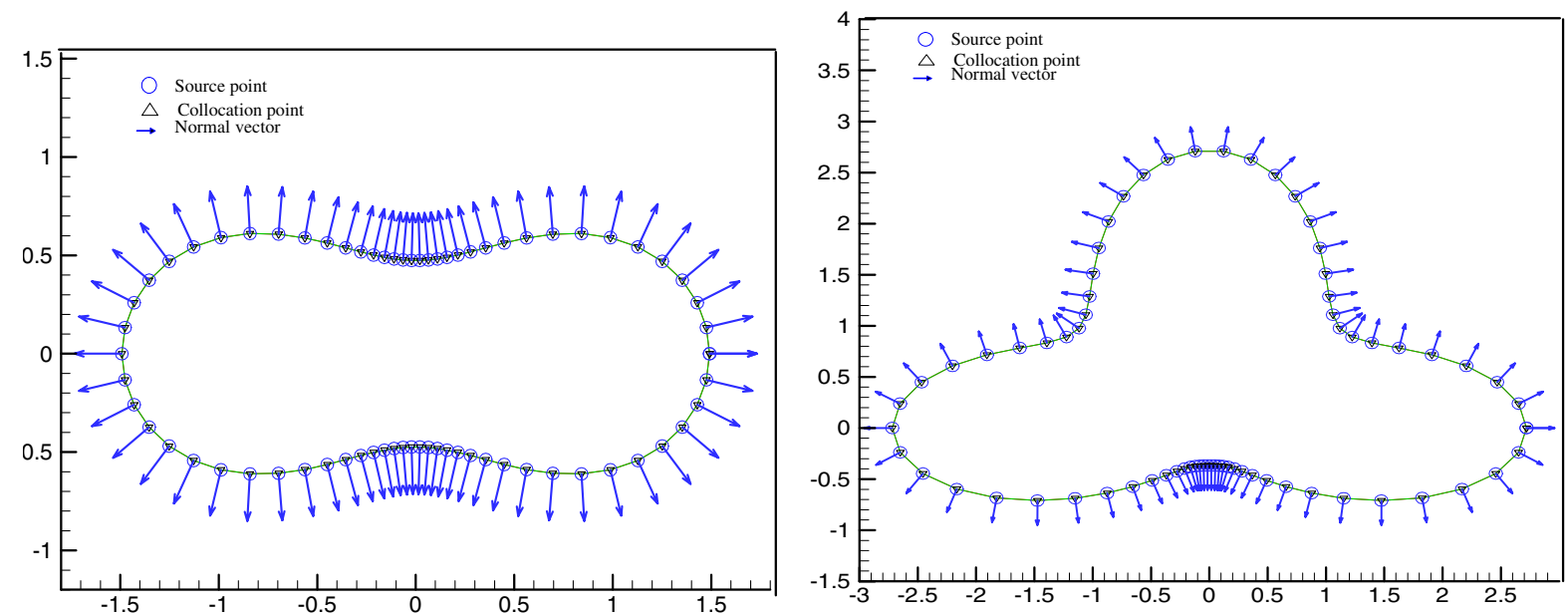

(a)

(b)

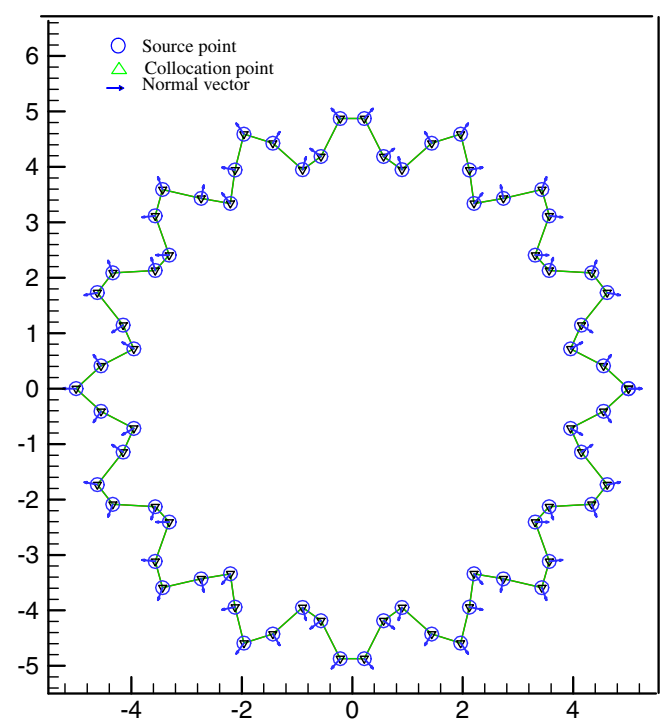

(c)

Fig. 21. The nodes distribution (70 nodes): (a) case 3.1, (b) case 3.2, (c) case 3.3. 


\subsection{Example 3: arbitrary domain cases (cases 3.1-3.3)}

In cases 3.1-3.3, the interior Dirichlet problems with peanut, armor-unit and gear wheel shapes for more complex boundaries are undertaken. Figs. 20 and 21, respectively, depict the geometry sketch and the node distributions of these three problems. The BCs and analytical solutions for the chosen
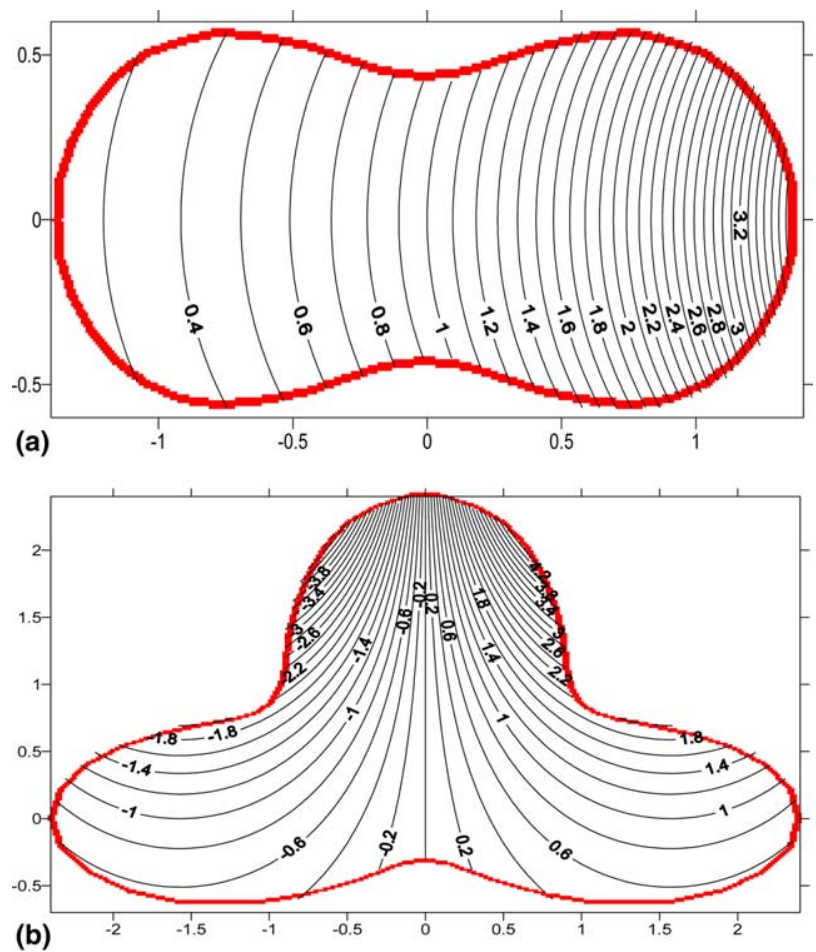

(b)

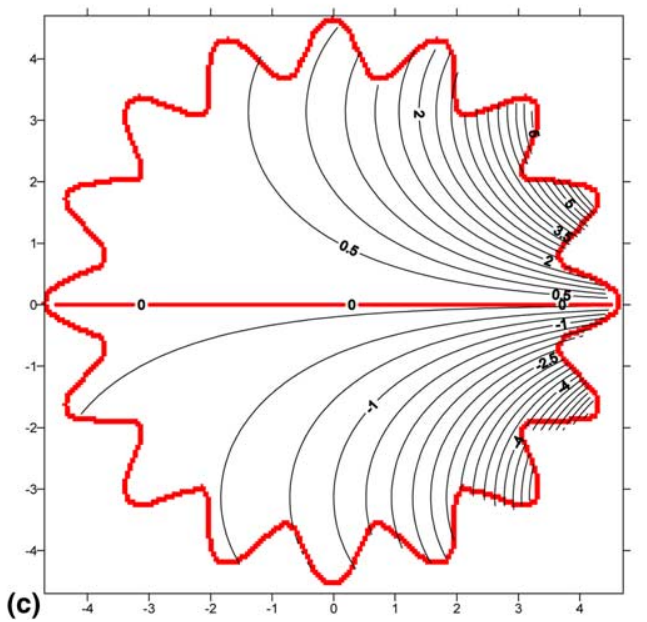

Fig. 22. The exact field solutions: (a) case 3.1, (b) case 3.2, (c) case 3.3 . 
problems are also shown in Fig. 20. The three field potential analytical solutions are plotted in Figs. 22(a)-(c), respectively, while Figs. 23(a)-(c) plot the three numerical results by using the proposed novel method. Fig. 23 shows good numerical results are obtained after comparing with the exact solutions.
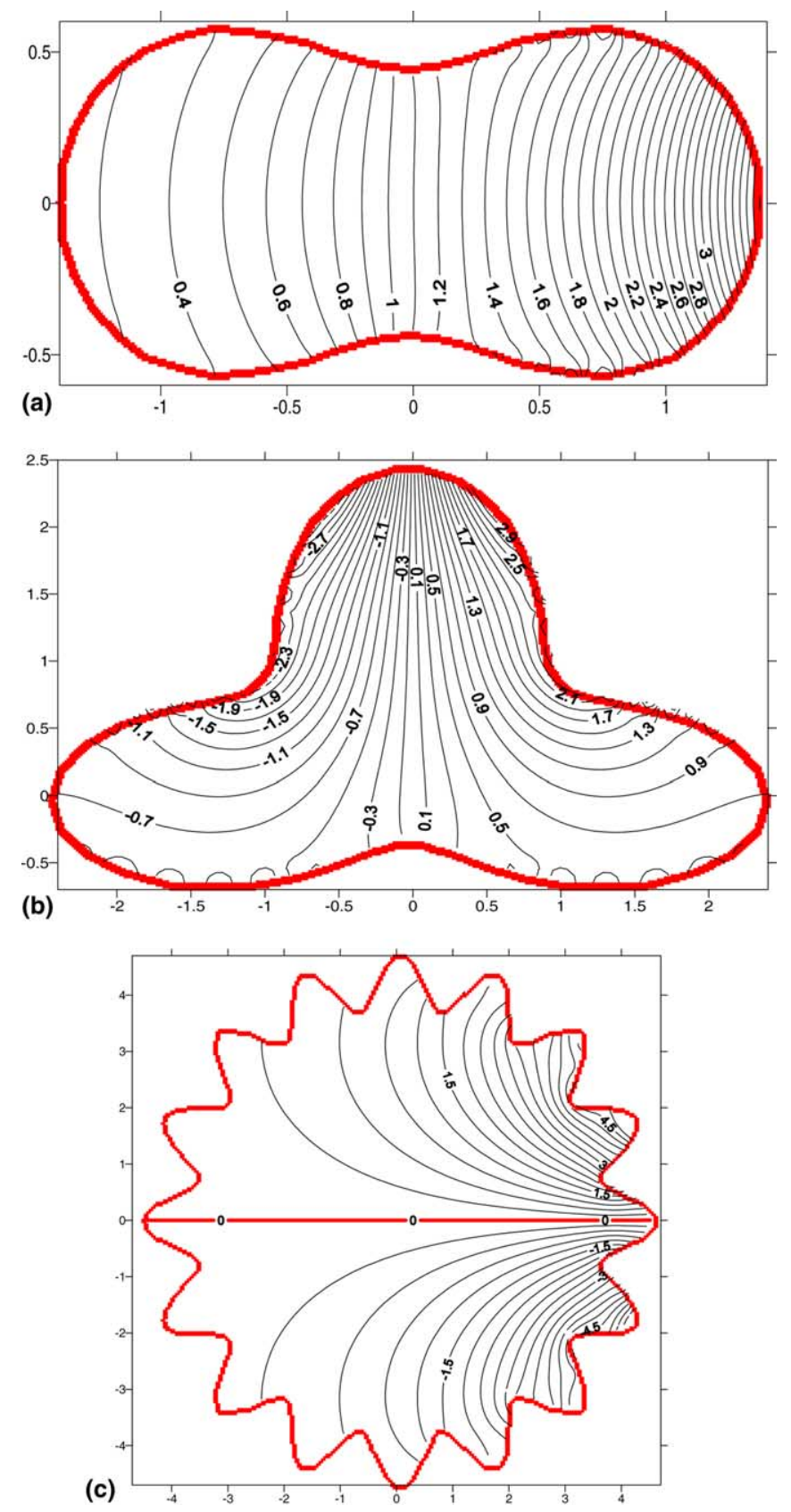

Fig. 23. The field solutions by using the novel meshless method (70 nodes): (a) case 3.1, (b) case 3.2, (c) case 3.3 . 


\section{Conclusions}

In this study, we implement a novel meshless method to solve the Laplace problems for arbitrary domains subject to the Dirichlet, Neumann and mixed-type BCs. Only the boundary nodes on the real boundary are required. The major difficulty of the coincidence of the source and collocation points in the conventional MFS is then circumvented. Furthermore, the controversy of the artificial (off-set) boundary outside the physical domain by using the conventional MFS no longer exists. Although it results in the singularity and hypersingularity due to using the double layer potential, the finite values of the diagonal terms for the influence matrices have been extracted out by the proposed desingularization technique to regularize the singularity and hypersingularity of the kernel functions. The ill-posed influence matrices generated by using the conventional MFS are eliminated when using the off-set boundary far from the real boundary. The numerical results were obtained by using the developed program for three category examples with different BCs and shapes of domain. Solutions were compared very well with the analytical solutions or other numerical methods such as BEM and conventional MFS.

\section{Acknowledgments}

Financial support from the National Science Council of Taiwan is gratefully acknowledged. It is granted to the National Taiwan University under Grant No. NSC-92-2281-E-002-020. We thank the reviewers' very constructive comments about this paper.

\section{Appendix A. The detail derivations of Eqs. (20) and (21)}

The null-fields of the boundary integral equations (BIEs) based on the direct method are

$$
\begin{aligned}
& 0=\int_{B} \frac{\partial \Phi^{(\mathrm{e})}\left(s, x^{i}\right)}{\partial n_{s}} \phi(s) \mathrm{d} B(s)-\int_{B} \Phi^{(\mathrm{e})}\left(s, x^{i}\right) \frac{\partial \phi(s)}{\partial n_{s}} \mathrm{~d} B(s), \quad x^{i} \in D^{e}, \\
& 0=\int_{B} \frac{\partial^{2} \Phi^{(\mathrm{e})}\left(s, x^{i}\right)}{\partial n_{s} \partial n_{x^{i}}} \phi(s) \mathrm{d} B(s)-\int_{B} \frac{\partial \Phi^{(\mathrm{e})}\left(s, x^{i}\right)}{\partial n_{x^{i}}} \frac{\partial \phi(s)}{\partial n_{s}} \mathrm{~d} B(s), \quad x^{i} \in D^{e},
\end{aligned}
$$

where the superscript (e) denotes the exterior domain, $\Phi$ is the single layer potential, and is equal to $\ln \left(\bar{r}_{i j}\right)$. Let $\frac{\partial \Phi^{(\mathrm{e})}\left(s, x^{i}\right)}{\partial n_{s}}=A^{(\mathrm{e})}\left(s, x^{i}\right)$, and $\frac{\partial^{2} \Phi^{(\mathrm{e})}\left(s, x^{i}\right)}{\partial n_{s} \partial n_{x^{i}}}=B^{(\mathrm{e})}\left(s, x^{i}\right)$. By employing the simple test method $\left(\partial \phi(s) / \partial n_{s}=0\right.$ when $\phi(s)=1$ ), we can write Eqs. (A.1) and (A.2) as follows:

$$
\begin{aligned}
& \int_{B} A^{(\mathrm{e})}\left(s, x^{i}\right) \mathrm{d} B(s)=0, \quad x^{i} \in D^{e}, \\
& \int_{B} B^{(\mathrm{e})}\left(s, x^{i}\right) \mathrm{d} B(s)=0, \quad x^{i} \in D^{e} .
\end{aligned}
$$

When the field point $x^{i}$ approaches the boundary, we can discretize Eqs. (A.3) and (A.4) and as follows:

$$
\sum_{j=1}^{N} A^{(\mathrm{e})}\left(s^{j}, x^{i}\right) \ell^{j}=0, \quad x^{i} \in B,
$$




$$
\sum_{j=1}^{N} B^{(\mathrm{e})}\left(s^{j}, x^{i}\right) \ell^{j}=0, \quad x^{i} \in B,
$$

where $\ell^{j}$ is the half of distance of the $(j-1)$ th source point and the $(j+1)$ th source point. When the distribution of nodes is uniform, we are able to reduce Eqs. (A.5) and (A.6) to the following

$$
\begin{aligned}
& \sum_{j=1}^{N} A^{(\mathrm{e})}\left(s^{j}, x^{i}\right)=0, \quad x^{i} \in B, \\
& \sum_{j=1}^{N} B^{(\mathrm{e})}\left(s^{j}, x^{i}\right)=0, \quad x^{i} \in B,
\end{aligned}
$$

where

$$
\begin{aligned}
& A^{(\mathrm{e})}\left(s^{j}, x^{i}\right)=\frac{\partial \Phi^{(\mathrm{e})}\left(s^{j}, x^{i}\right)}{\partial n_{s}}=\frac{n_{k} y_{k}}{\bar{r}_{i j}^{2}}, \\
& B^{(\mathrm{e})}\left(s^{j}, x^{i}\right)=\frac{\partial^{2} \Phi^{(\mathrm{e})}\left(s^{j}, x^{i}\right)}{\partial n_{s} \partial n_{x^{i}}}=2 \frac{y_{k} y_{l} n_{k} \bar{n}_{l}}{\bar{r}_{i j}^{4}}-\frac{n_{k} \bar{n}_{k}}{\bar{r}_{i j}^{2}},
\end{aligned}
$$

where $\bar{r}_{i j}=\left|s^{j}-x^{i}\right|, n_{k}$ is the $k$ th component of the outward normal vector at $s^{j} ; \bar{n}_{k}$ is the $k$ th component of the outward normal vector at $x^{i}$ and $y_{k}=x_{k}^{i}-s_{k}^{j}$. Eqs. (A.7) and (A.8) are Eqs. (20) and (21) in the text of Section 3; and Eqs. (A.9) and (A.10) are Eqs. (16) and (17) in the text of Section 2.

\section{Appendix B. Analytical derivation of diagonal coefficients of influence matrices for circular domain by using separable kernels and circulants}

By adopting the addition theorem [1], we can expand the two kernels in Eqs. (16) and (17) for exterior problems and also the corresponding two kernels for the interior problems into separable kernels which separate the field point, $x^{i}$, and source point, $s^{j}$, as follows:

$$
\begin{aligned}
& A\left(s^{j}, x^{i}\right)=\frac{\partial \ln \left(\bar{r}_{i j}\right)}{\partial r}=\frac{r-\rho \cos (\theta-\varphi)}{r^{2}+\rho^{2}-2 r \rho \cos (\theta-\varphi)}= \begin{cases}A^{(\mathrm{i})}\left(s^{j}, x^{i}\right)=\frac{1}{r}+\sum_{m=1}^{\infty} \frac{\rho^{m}}{r^{m+1}} \cos (m(\theta-\varphi)), & r>\rho, \\
A^{(\mathrm{e})}\left(s^{j}, x^{i}\right)=-\sum_{m=1}^{\infty} \frac{r^{m-1}}{\rho^{m}} \cos (m(\theta-\varphi)), & \rho>r,\end{cases} \\
& B\left(s^{j}, x^{i}\right)=\frac{\partial^{2} \ln \left(\bar{r}_{i j}\right)}{\partial \rho \partial r}=\frac{-2 r \rho+\left(r^{2}+\rho^{2}\right) \cos (\theta-\varphi)}{\left(r^{2}+\rho^{2}-2 r \rho \cos (\theta-\varphi)\right)^{2}}= \begin{cases}B^{(\mathrm{i})}\left(s^{j}, x^{i}\right)=\sum_{m=1}^{\infty} \frac{m \rho^{m-1}}{r^{m+1}} \cos (m(\theta-\varphi)), & r>\rho, \\
B^{(\mathrm{e})}\left(s^{j}, x^{i}\right)=\sum_{m=1}^{\infty} \frac{m r^{m-1}}{\rho^{m+1}} \cos (m(\theta-\varphi)), & \rho>r,\end{cases}
\end{aligned}
$$

where $s^{j}=(r, \theta)$ and $x^{i}=(\rho, \varphi)$ in the polar coordinates. The definitions of $r, \theta, \rho, \varphi$ for the interior and exterior problems are plotted in Figs. 1(c) and (d). Since the rotation symmetry is preserved for a circular boundary, the two influence matrices in Eqs. (6) and (7) are the circulants with the elements

$$
K_{i j}=K\left(r, \theta_{j} ; \rho, \varphi_{i}\right)
$$


where the kernel $K$ can be $A$ or $B$ in Eqs. (6) and (7) for the interior problems and Eqs. (12) and (13) for the exterior problems, $\theta_{j}, \varphi_{i}$ are the angles of source and collocation points, respectively. By superimposing $N$ lumped strength along the boundary, we have the following influence matrices:

$$
[K]=\left[\begin{array}{cccc}
k_{0} & k_{1} & \cdots & k_{N-1} \\
k_{N-1} & k_{0} & \cdots & k_{N-2} \\
\vdots & \vdots & \ddots & \vdots \\
k_{1} & k_{2} & \cdots & k_{0}
\end{array}\right]
$$

where the elements of the first row can be obtained by

$$
k_{j}=k\left(r, \theta_{j} ; \rho, 0\right),
$$

in which $\varphi=0$ is assigned without loss of generality. The matrix $[K]$ in Eq. (B.4) is found to be a circulant since the rotational symmetry for the influence coefficients is considered. By introducing the following bases for the circulants, $I,\left(C_{N}\right)^{1},\left(C_{N}\right)^{2}, \ldots$, and $\left(C_{N}\right)^{N-1}$, we can expand $[K]$ into

$$
[K]=k_{0} I+k_{1}\left(C_{N}\right)^{1}+k_{2}\left(C_{N}\right)^{2}+\cdots+k_{N-1}\left(C_{N}\right)^{N-1},
$$

where $I$ is an unit matrix and

$$
C_{N}=\left[\begin{array}{cccccc}
0 & 1 & 0 & \cdots & 0 & 0 \\
0 & 0 & 1 & \cdots & 0 & 0 \\
\vdots & \vdots & \vdots & \ddots & \vdots & \vdots \\
1 & 0 & 0 & \cdots & 0 & 0
\end{array}\right]_{N \times N}
$$

Based on the circulant theory [4], the eigenvalues for the influence matrix, $[K]$, are found as follows:

$$
\lambda_{l}=k_{0}+k_{1} \tau_{l}+k_{2}\left(\tau_{l}\right)^{2}+\cdots+k_{N-1}\left(\tau_{l}\right)^{N-1}, \quad l=0,1,2, \ldots, N-1,
$$

where $\lambda_{l}$ and $\tau_{l}$ are the eigenvalues for $[K]$ and $\left[C_{N}\right]$, respectively. It is easily found that the eigenvalues $\tau_{l}$ for the circulant $\left[C_{N}\right]$ are the roots for $\tau^{N}=1$ as shown below:

$$
\tau_{l}=\mathrm{e}^{\mathrm{i} 2 \pi l}, \quad l=0,1,2, \ldots, N-1
$$

Substituting Eq. (B.9) into Eq. (B.8), we have

$$
\lambda_{l}=\sum_{m=0}^{N-1} k_{m} \tau_{l}^{m}=\sum_{m=0}^{N-1} k_{m} \mathrm{e}^{\mathrm{i} \frac{2 \pi m l}{N}}, \quad l=0,1,2, \ldots, N-1 .
$$

According to the definition for $k_{m}$ in Eq. (B.5), we obtain

$$
k_{m}=k_{N-m}, \quad m=0,1,2, \ldots, N-1 .
$$

Substitution of Eq. (B.11) into Eq. (B.10) it yields

$$
\lambda_{l}=\sum_{m=0}^{N-1} k_{m} \cos \left(\frac{2 \pi m l}{N}\right), \quad l=0,1,2, \ldots, N-1 .
$$

By setting $\varphi=0$ without loss of generality, the Riemann sum of infinite terms reduces to the following integral

$$
\lambda_{l}=\frac{1}{\Delta \theta} \lim _{N \rightarrow \infty} \sum_{m=0}^{N-1} K(m \Delta \theta, 0) \cos (m l \Delta \theta) \Delta \theta \approx \frac{N}{2 \pi} \int_{0}^{2 \pi} \cos (l \theta) K(\theta, 0) \mathrm{d} \theta,
$$


where $\Delta \theta=\frac{2 \pi}{N}$.

\section{B.1. Interior problem}

By employing the separable kernel $A^{(\mathrm{i})}\left(s^{j}, x^{i}\right)$ for interior problem $(r>\rho)$ in Eq. (B.1) and the orthogonal conditions, Eq. (B.13) reduces to

$$
v_{l}^{(\mathrm{i})}= \begin{cases}\frac{N}{r}, & l=0, \\ \frac{N}{2 r}, & l=0,1,2, \ldots, N-1 .\end{cases}
$$

Similarly, we have

$$
\delta_{l}^{(\mathrm{i})}= \begin{cases}0, & l=0 \\ \frac{N|l|}{2 r^{2}}, & l=0,1,2, \ldots, N-1,\end{cases}
$$

where $v_{l}^{(\mathrm{i})}$ and $\delta_{l}^{(\mathrm{i})}$ are the eigenvalues of $\left[A^{(\mathrm{i})}\right]$ and $\left[B^{(\mathrm{i})}\right]$ matrices, respectively. By employing the invariant property for the influence matrices, the first invariant is the sum of all the eigenvalues. The diagonal coefficients for the two matrices for the interior problem are obtained by adding all the eigenvalues and can be shown below:

$$
\begin{aligned}
N a_{j j} & =\sum_{m=0}^{N-1} v_{m}^{(\mathrm{i})} \quad(j \text { no sum }), \\
N b_{j j} & =\sum_{m=0}^{N-1} \delta_{m}^{(\mathrm{i})} .
\end{aligned}
$$

Hence, the diagonal elements are easily determined from the first invariant as follows:

$$
\begin{aligned}
& a_{j j}=\frac{N+1}{2 r} \approx \frac{\pi}{\frac{2 \pi r}{N}}, \quad N \gg 1, \\
& b_{j j}=\frac{N(N-1)}{4 r^{2}} \approx \frac{\pi^{2}}{\left(\frac{2 \pi r}{N}\right)^{2}}, \quad N \gg 1 .
\end{aligned}
$$

\section{B.2. Exterior problem}

Similarly, we have the diagonal terms of the influence matrices for the exterior problem as follows:

$$
\begin{aligned}
& \bar{a}_{j j}=\frac{N-1}{2 r} \approx \frac{\pi}{\frac{2 \pi r}{N}}, \quad N \gg 1, \\
& \bar{b}_{j j}=\frac{N(N-1)}{4 r^{2}} \approx \frac{\pi^{2}}{\left(\frac{2 \pi r}{N}\right)^{2}}, \quad N \gg 1 .
\end{aligned}
$$

The properties of the influence matrices for interior and exterior problems are shown in Table 1 .

\section{References}

[1] M. Abramowitz, I.A. Stegun, Handbook of Mathematical Functions with Formulation, Graphs and Mathematical Tables, Dover, New York, 1972. 
[2] T. Belytschko, L. Gu, Y. Lu, Fracture and crack growth by element-free Galerkin methods, Model. Simul. Mater. Sci. Eng. 2 (1994) 519-534.

[3] J.T. Chen, S.R. Kuo, K.H. Chen, Y.C. Cheng, Comments on vibration analysis of arbitrary shaped membranes using nondimensional dynamic influence function, J. Sound Vibr. 235 (1) (2000) 156-171.

[4] J.T. Chen, M.H. Chang, K.H. Chen, S.R. Lin, The boundary collocation method with meshless concept for acoustic eigenanalysis of two-dimensional cavities using radial basis function, J. Sound Vibr. 257 (4) (2002) 667-711.

[5] J.T. Chen, M.H. Chang, K.H. Chen, Boundary collocation method for acoustic eigenanalysis of three-dimensional cavities using radial basis function, Comput. Mech. 29 (2002) 392-408.

[6] J.T. Chen, I.L. Chen, C.S. Wu, On the equivalence of MFS and Trefftz method for Laplace problems, in: Proceedings of the Global Chinese Workshop on Boundary Element and Meshless Method, Hebei, China, 2003.

[7] J.T. Chen, I.L. Chen, K.H. Chen, Y.T. Yeh, Y.T. Lee, A meshless method for free vibration of arbitrarily shaped plates with clamped boundaries using radial basis function, Eng. Anal. Bound Elem. 28 (2004) 535-545.

[8] W. Chen, M. Tanaka, A meshfree, integration-free and boundary-only RBF technique, Comput. Math. Appl. 43 (2002) 379-391.

[9] W. Chen, Y.C. Hon, Numerical investigation on convergence of boundary knot method in the analysis of homogeneous Helmholtz, modified Helmholtz and convection-diffusion problems, Comput. Methods Appl. Mech. Eng. 192 (2003) $1859-1875$.

[10] A.H.D. Cheng, D.L. Young, C.C. Tsai, The solution of Poisson's equation by iterative DRBEM using compactly supported, positive definite radial basis function, Eng. Anal. Bound. Elem. 24 (7) (2000) 549-557.

[11] G. Fairweather, A. Karageorghis, The method of fundamental solutions for elliptic boundary value problems, Adv. Comput. Math. 9 (1998) 69-95.

[12] R.A. Gingold, J.J. Maraghan, Smoothed particle hydrodynamics: theory and applications to non-spherical stars, Man. Not. Astron. Soc. 181 (1977) 375-389.

[13] W.S. Hwang, L.P. Hung, C.H. Ko, Non-singular boundary integral formulations for plane interior potential problems, Int. J. Numer. Meth. Eng. 53 (2002) 1751-1762.

[14] S.W. Kang, J.M. Lee, Y.J. Kang, Vibration analysis of arbitrary shaped membranes using non-dimensional dynamic influence function, J. Sound Vibr. 221 (1) (1999) 117-132.

[15] S.W. Kang, J.M. Lee, Application of free vibration analysis of membranes using the non-dimensional dynamic influence function, J. Sound Vibr. 234 (3) (2000) 455-470.

[16] W.K. Liu, S. Jun, S. Li, J. Adee, T. Belytschko, Reproducing kernel particle methods for structural dynamics, Int. J. Numer. Meth. Eng. 38 (1995) 1655-1679.

[17] M.A. Tournour, N. Atalla, Efficient evaluation of the acoustic radiation using multipole expansion, Int. J. Numer. Meth. Eng. 46 (1999) 825-837.

[18] C.C. Tsai, Meshless numerical methods and their engineering applications, Ph.D. Dissertation, National Taiwan University, Taipei, Taiwan, 2002.

[19] C.C. Tsai, D.L. Young, A.H.D. Cheng, Meshless BEM for three-dimensional Stokes flows, Computer Modeling in Engineering and Science (CMES) 3 (2002) 117-128.

[20] Y.S. Smyrlis, A. Karageorghis, Some aspects of the method of fundamental solutions for certain harmonic problems, J. Scientific Comput. 16 (3) (2001) 341-371.

[21] D.L. Young, C.C. Tsai, T.I. Eldho, A.H.D. Cheng, Solution of Stokes flow using an iterative DRBEM based on compactlysupported, positive definite radial basis function, Comput. Math. Appl. 43 (2002) 607-619.

[22] Y.X. Mukherjee, S. Mukherjee, The boundary node method for potential problems, Int. J. Numer. Meth. Eng. 40 (1997) $797-815$.

[23] J.M. Zhang, M. Tanaka, T. Matsumoto, Meshless analysis of potential problems in three dimensions with the hybrid boundary node method, Int. J. Numer. Meth. Eng. 59 (2004) 1147-1160.

[24] J.M. Zhang, Z.H. Yao, H. Li, A hybrid boundary node method, Int. J. Numer. Meth. Eng. 53 (2002) $751-763$. 